\title{
Measurement of $\Gamma\left(Z^{0} \rightarrow \mathbf{b} \bar{b}\right) / \Gamma\left(Z^{0} \rightarrow\right.$ hadrons $)$ using a Double Tagging Method
}

\author{
The OPAL Collaboration
}

\begin{abstract}
The fraction of $\mathrm{Z}^{0} \rightarrow \mathrm{b} \overline{\mathrm{b}}$ events in hadronic $\mathrm{Z}^{0}$ decays has been measured using the data collected by OPAL in 1992 and 1993. The presence of electrons or muons from semileptonic decays of bottom hadrons and the detection of bottom hadron decay vertices were used together to obtain an event sample enriched in $Z^{0} \rightarrow b \bar{b}$ decays. To reduce the systematic error on the measurement of the $\mathrm{Z}^{0} \rightarrow \mathrm{b} \overline{\mathrm{b}}$ fraction, the efficiency of the $\mathrm{b} \overline{\mathrm{b}}$ event tagging was obtained from the data by comparing the numbers of events having a bottom signature in either one or both thrust hemispheres. A value of

$$
\frac{\Gamma\left(Z^{0} \rightarrow b \bar{b}\right)}{\Gamma\left(Z^{0} \rightarrow \text { hadrons }\right)}=0.2171 \pm 0.0021 \pm 0.0021
$$

was obtained, where the first error is statistical and the second systematic. The uncertainty on the decay width $\Gamma\left(\mathrm{Z}^{0} \rightarrow \mathrm{c} \overline{\mathrm{c}}\right)$ is not included in these errors. A fractional variation of this width by $\pm 8 \%$ about its Standard Model prediction would result in a variation of the measured $Z^{0} \rightarrow b \bar{b}$ fraction of $\mp 0.0015$.
\end{abstract}




\section{The OPAL Collaboration}

R. Akers ${ }^{16}$, G. Alexander ${ }^{23}$, J. Allison ${ }^{16}$, K.J. Anderson ${ }^{9}$, S. Arcelli $^{2}$, S. Asai $^{24}$, A. Astbury ${ }^{28}$, D. Axen ${ }^{29}$, G. Azuelos ${ }^{18, a}$, A.H. Ball ${ }^{17}$, E.Barberio ${ }^{26}$, R.J. Barlow ${ }^{16}$, R.Bartoldus ${ }^{3}$, J.R.Batley ${ }^{5}$, G. Beaudoin ${ }^{18}$, A.Beck ${ }^{23}$, G.A.Beck ${ }^{13}$, J.Becker ${ }^{10}$, C. Beeston ${ }^{16}$, T. Behnke ${ }^{27}$, K.W.Bell ${ }^{20}$, G.Bella ${ }^{23}$,

P. Bentkowski ${ }^{18}$, S. Bentvelsen ${ }^{8}$, P. Berlich ${ }^{10}$, S.Bethke ${ }^{32}$, O. Biebel ${ }^{32}$, I.J. Bloodworth ${ }^{1}$, P. Bock ${ }^{11}$, H.M. Bosch ${ }^{11}$, M. Boutemeur ${ }^{18}$, S.Braibant ${ }^{12}$, P. Bright-Thomas ${ }^{25}$, R.M. Brown ${ }^{20}$, A. Buijs ${ }^{8}$,

H.J.Burckhart ${ }^{8}$, C.Burgard ${ }^{27}$, P. Capiluppi ${ }^{2}$, R.K. Carnegie ${ }^{6}$, A.A. Carter ${ }^{13}$, J.R. Carter ${ }^{5}$,

C.Y.Chang ${ }^{17}$, C. Charlesworth ${ }^{6}$, D.G. Charlton ${ }^{8}$, S.L. Chu ${ }^{4}$, P.E.L.Clarke ${ }^{15}$, J.C. Clayton ${ }^{1}$, S.G.Clowes ${ }^{16}$, I. Cohen ${ }^{23}$, J.E. Conboy ${ }^{15}$, M.Coupland ${ }^{14}$, M.Cuffiani ${ }^{2}$, S. Dado ${ }^{22}$, C. Dallapiccola ${ }^{17}$, G.M.Dallavalle ${ }^{2}$, C.Darling ${ }^{31}$, S. De Jong ${ }^{13}$, H.Deng ${ }^{17}$, M. Dittmar ${ }^{4}$, M.S. Dixit ${ }^{7}$, E. do Couto e Silva $^{12}$, J.E. Duboscq ${ }^{8}$, E. Duchovni ${ }^{26}$, G. Duckeck ${ }^{8}$, I.P.Duerdoth ${ }^{16}$, U.C.Dunwoody ${ }^{5}$, P.A.Elcombe ${ }^{5}$, P.G.Estabrooks ${ }^{6}$, E.Etzion ${ }^{23}$, H.G.Evans ${ }^{9}$, F.Fabbri ${ }^{2}$, B. Fabbro ${ }^{21}$, M. Fanti ${ }^{2}$, M. Fierro ${ }^{2}$, M. Fincke-Keeler ${ }^{28}$, H.M. Fischer ${ }^{3}$, P. Fischer ${ }^{3}$, R. Folman ${ }^{26}$, D.G. Fong ${ }^{17}$, M. Foucher ${ }^{17}$, H. Fukui ${ }^{24}$,

A.Fürtjes ${ }^{8}$, P. Gagnon ${ }^{6}$, A. Gaidot ${ }^{21}$, J.W. Gary ${ }^{4}$, J. Gascon $^{18}$, N.I. Geddes ${ }^{20}$, C. Geich-Gimbel ${ }^{3}$, S.W. Gensler ${ }^{9}$, F.X. Gentit ${ }^{21}$, T. Geralis ${ }^{20}$, G. Giacomelli ${ }^{2}$, P. Giacomelli ${ }^{4}$, R. Giacomelli ${ }^{2}$, V. Gibson ${ }^{5}$, W.R. Gibson ${ }^{13}$, J.D. Gillies ${ }^{20}$, J. Goldberg ${ }^{22}$, D.M. Gingrich ${ }^{30, a}$, M.J. Goodrick ${ }^{5}$, W. Gorn ${ }^{4}$, C. Grandi ${ }^{2}$, P. Grannis ${ }^{8}$, E. Gross ${ }^{26}$, J. Hagemann ${ }^{27}$, G.G.Hanson ${ }^{12}$, M. Hansroul ${ }^{8}$, C.K. Hargrove ${ }^{7}$, J. Hart ${ }^{8}$, P.A.Hart ${ }^{9}$, M. Hauschild ${ }^{8}$, C.M.Hawkes ${ }^{8}$, E. Heflin ${ }^{4}$, R.J.Hemingway ${ }^{6}$, G.Herten ${ }^{10}$, R.D.Heuer ${ }^{8}$, J.C.Hill ${ }^{5}$, S.J. Hillier ${ }^{8}$, T. Hilse ${ }^{10}$, D.A.Hinshaw ${ }^{18}$, P.R. Hobson ${ }^{25}$, D. Hochman ${ }^{26}$, A. Höcker ${ }^{3}$, R.J.Homer ${ }^{1}$, A.K.Honma ${ }^{28, a}$, R.E.Hughes-Jones ${ }^{16}$, R. Humbert ${ }^{10}$, P. Igo-Kemenes ${ }^{11}$, H. Thssen ${ }^{11}$, D.C.Imrie ${ }^{25}$, A. Jawahery ${ }^{17}$, P.W. Jeffreys ${ }^{20}$, H. Jeremie ${ }^{18}$, M. Jimack ${ }^{1}$, M. Jones ${ }^{6}$, R.W.L. Jones ${ }^{8}$, P. Jovanovic ${ }^{1}$, C. Jui ${ }^{4}$, D. Karlen ${ }^{6}$, K. Kawagoe ${ }^{24}$, T. Kawamoto ${ }^{24}$, R.K. Keeler ${ }^{28}$, R.G.Kellogg ${ }^{17}$, B.W.Kennedy ${ }^{20}$, B.King ${ }^{8}$, J.King ${ }^{13}$, S. Kluth ${ }^{5}$, T. Kobayashi ${ }^{24}$, M.Kobel ${ }^{10}$, D.S.Koetke ${ }^{8}$, T.P.Kokott ${ }^{3}$, S.Komamiya ${ }^{24}$, R.Kowalewski ${ }^{8}$, R. Howard ${ }^{29}$, P. Krieger ${ }^{6}$, J. von Krogh $^{11}$, P.Kyberd ${ }^{13}$, G.D. Lafferty ${ }^{16}$, H. Lafoux ${ }^{8}$, R. Lahmann ${ }^{17}$, J. Lauber ${ }^{8}$, J.G. Layter ${ }^{4}$,

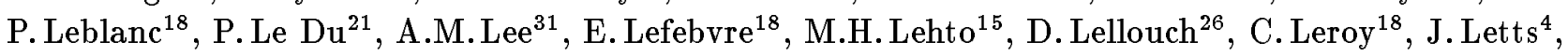
L. Levinson ${ }^{26}$, Z. Li $^{12}$, F. Liu $^{29}$, S.L.Lloyd ${ }^{13}$, F.K.Loebinger ${ }^{16}$, G.D.Long ${ }^{17}$, B. Lorazo ${ }^{18}$, M.J. Losty ${ }^{7}$,

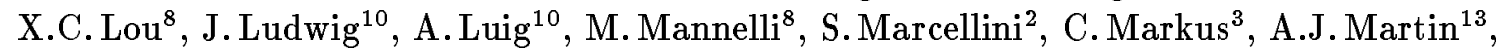
J.P. Martin ${ }^{18}$, T. Mashimo ${ }^{24}$, P. Mättig ${ }^{3}$, U. Maur ${ }^{3}$, J.McKenna ${ }^{29}$, T.J.McMahon ${ }^{1}$, A.I.McNab ${ }^{13}$,

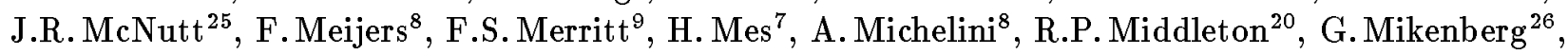
J. Mildenberger ${ }^{6}$, D.J. Miller ${ }^{15}$, R. Mir $^{26}$, W.Mohr ${ }^{10}$, C.Moisan ${ }^{18}$, A.Montanari ${ }^{2}$, T.Mori ${ }^{24}$, M. Morii' ${ }^{24}$, U.Müller ${ }^{3}$, B. Nellen ${ }^{3}$, B. Nijjhar ${ }^{16}$, S.W. O'Neale ${ }^{1}$, F.G.Oakham ${ }^{7}$, F. Odorici ${ }^{2}$, H.O. Ogren ${ }^{12}$, C.J. Oram ${ }^{28, a}$, M.J. Oreglia ${ }^{9}$, S. Orito ${ }^{24}$, J.P. Pansart ${ }^{21}$, G.N.Patrick ${ }^{20}$, M.J.Pearce ${ }^{1}$, P.Pfister ${ }^{10}$, P.D.Phillips ${ }^{16}$, J.E.Pilcher ${ }^{9}$, J.Pinfold ${ }^{30}$, D. Pitman $^{28}$, D.E.Plane ${ }^{8}$, P. Poffenberger ${ }^{28}$, B. Poli $^{2}$, A. Posthaus $^{3}$, T.W.Pritchard ${ }^{13}$, H.Przysiezniak ${ }^{18}$, M.W.Redmond ${ }^{8}$, D.L. Rees ${ }^{8}$, D. Rigby ${ }^{1}$, M. Rison ${ }^{5}$, S.A.Robins ${ }^{13}$, D. Robinson ${ }^{5}$, J.M.Roney ${ }^{28}$, E. Ros $^{8}$, S.Rossberg ${ }^{10}$, A.M.Rossi ${ }^{2}$, M.Rosvick ${ }^{28}$, P.Routenburg ${ }^{30}$, Y.Rozen ${ }^{8}$, K. Runge ${ }^{10}$, O.Runolfsson ${ }^{8}$, D.R.Rust ${ }^{12}$, M. Sasaki ${ }^{24}$,

C.Sbarra ${ }^{2}$, A.D. Schaile ${ }^{8}$, O.Schaile ${ }^{10}$, F. Scharf ${ }^{3}$, P.Scharff-Hansen ${ }^{8}$, P. Schenk ${ }^{4}$, B. Schmitt ${ }^{3}$, H.von der Schmitt ${ }^{11}$, M. Schröder ${ }^{12}$, H.C.Schultz-Coulon ${ }^{10}$, P. Schütz ${ }^{3}$, M. Schulz ${ }^{8}$, C. Schwick ${ }^{27}$,

J.Schwiening ${ }^{3}$, W.G.Scott ${ }^{20}$, M.Settles ${ }^{12}$, T.G.Shears ${ }^{5}$, B.C.Shen ${ }^{4}$, C.H.Shepherd-Themistocleous ${ }^{7}$, P.Sherwood ${ }^{15}$, G.P.Siroli ${ }^{2}$, A.Skillman ${ }^{16}$, A.Skuja ${ }^{17}$, A.M.Smith ${ }^{8}$, T.J. Smith ${ }^{28}$, G.A.Snow ${ }^{17}$, R. Sobie ${ }^{28}$, R.W.Springer ${ }^{17}$, M.Sproston ${ }^{20}$, A.Stahl ${ }^{3}$, C.Stegmann ${ }^{10}$, K.Stephens ${ }^{16}$, J.Steuerer ${ }^{28}$, B.Stockhausen ${ }^{3}$, R.Ströhmer ${ }^{11}$, D.Strom ${ }^{19}$, P.Szymanski ${ }^{20}$, H. Takeda ${ }^{24}$, T. Takeshita ${ }^{24}$, S. Tarem ${ }^{26}$, M. Tecchio ${ }^{9}$, P. Teixeira-Dias ${ }^{11}$, N. Tesch ${ }^{3}$, M.A.Thomson ${ }^{15}$, S. Towers ${ }^{6}$, T. Tsukamoto ${ }^{24}$,

M.F. Turner-Watson ${ }^{8}$, D. Van den plas ${ }^{18}$, R. Van Kooten $^{12}$, G. Vasseur ${ }^{21}$, M. Vincter ${ }^{28}$, A. Wagner ${ }^{27}$,

D.L. Wagner ${ }^{9}$, C.P. Ward ${ }^{5}$, D.R. Ward ${ }^{5}$, J.J. Ward $^{15}$, P.M. Watkins ${ }^{1}$, A.T. Watson ${ }^{1}$, N.K. Watson ${ }^{7}$,

P. Weber ${ }^{6}$, P.S. Wells ${ }^{8}$, N. Wermes ${ }^{3}$, B.Wilkens ${ }^{10}$, G.W.Wilson ${ }^{4}$, J.A.Wilson ${ }^{1}$, V-H. Winterer ${ }^{10}$,

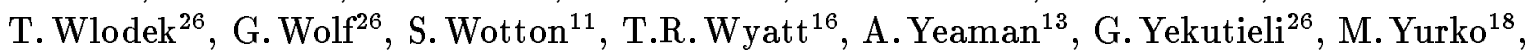
W. Zeuner ${ }^{8}$, G.T.Zorn ${ }^{17}$. 
${ }^{1}$ School of Physics and Space Research, University of Birmingham, Birmingham B15 2TT, UK

${ }^{2}$ Dipartimento di Fisica dell' Università di Bologna and INFN, I-40126 Bologna, Italy

${ }^{3}$ Physikalisches Institut, Universität Bonn, D-53115 Bonn, Germany

${ }^{4}$ Department of Physics, University of California, Riverside CA 92521, USA

${ }^{5}$ Cavendish Laboratory, Cambridge CB3 0HE, UK

${ }^{6}$ Carleton University, Department of Physics, Colonel By Drive, Ottawa, Ontario K1S 5B6, Canada

${ }^{7}$ Centre for Research in Particle Physics, Carleton University, Ottawa, Ontario K1S 5B6, Canada

${ }^{8}$ CERN, European Organisation for Particle Physics, CH-1211 Geneva 23, Switzerland

${ }^{9}$ Enrico Fermi Institute and Department of Physics, University of Chicago, Chicago IL 60637, USA

${ }^{10}$ Fakultät für Physik, Albert Ludwigs Universität, D-79104 Freiburg, Germany

${ }^{11}$ Physikalisches Institut, Universität Heidelberg, D-69120 Heidelberg, Germany

${ }^{12}$ Indiana University, Department of Physics, Swain Hall West 117, Bloomington IN 47405, USA

${ }^{13}$ Queen Mary and Westfield College, University of London, London E1 4NS, UK

${ }^{14}$ Birkbeck College, London WC1E 7HV, UK

${ }^{15}$ University College London, London WC1E 6BT, UK

${ }^{16}$ Department of Physics, Schuster Laboratory, The University, Manchester M13 9PL, UK

${ }^{17}$ Department of Physics, University of Maryland, College Park, MD 20742, USA

${ }^{18}$ Laboratoire de Physique Nucléaire, Université de Montréal, Montréal, Quebec H3C 3J7, Canada

${ }^{19}$ University of Oregon, Department of Physics, Eugene OR 97403, USA

${ }^{20}$ Rutherford Appleton Laboratory, Chilton, Didcot, Oxfordshire OX11 0QX, UK

${ }^{21}$ CEA, DAPNIA/SPP, CE-Saclay, F-91191 Gif-sur-Yvette, France

${ }^{22}$ Department of Physics, Technion-Israel Institute of Technology, Haifa 32000, Israel

${ }^{23}$ Department of Physics and Astronomy, Tel Aviv University, Tel Aviv 69978, Israel

${ }^{24}$ International Centre for Elementary Particle Physics and Department of Physics, University of Tokyo, Tokyo 113, and Kobe University, Kobe 657, Japan

${ }^{25}$ Brunel University, Uxbridge, Middlesex UB8 3PH, UK

${ }^{26}$ Particle Physics Department, Weizmann Institute of Science, Rehovot 76100, Israel

${ }^{27}$ Universität Hamburg/DESY, II Institut für Experimental Physik, Notkestrasse 85, D-22607 Hamburg, Germany

${ }^{28}$ University of Victoria, Department of Physics, P O Box 3055, Victoria BC V8W 3P6, Canada

${ }^{29}$ University of British Columbia, Department of Physics, Vancouver BC V6T 1Z1, Canada

${ }^{30}$ University of Alberta, Department of Physics, Edmonton AB T6G 2J1, Canada

${ }^{31}$ Duke University, Dept of Physics, Durham, NC 27708-0305, USA

${ }^{32}$ Technische Hochschule Aachen, III Physikalisches Institut, Sommerfeldstrasse 26-28, D-52056 Aachen, Germany

${ }^{a}$ Also at TRIUMF, Vancouver, Canada V6T $2 \mathrm{~A} 3$ 


\section{Introduction}

The partial width for the decay $\mathrm{Z}^{0} \rightarrow \mathrm{b} \overline{\mathrm{b}}$ is of special interest in the Standard Model. Electroweak corrections involving the top quark affect the $\mathrm{Z}^{0} \rightarrow \mathrm{b} \overline{\mathrm{b}}$ width, $\Gamma_{\mathrm{b}}$, differently from the widths for lighter quarks. ${ }^{1}$ This results in a reduced dependence of $\Gamma_{\mathrm{b}}$ on the top quark mass, $m_{\mathrm{top}}$, providing the possibility of a stringent and $m_{\mathrm{top}}$-independent test of the Standard Model [1]. On the other hand, the fraction

$$
\frac{\Gamma\left(\mathrm{Z}^{0} \rightarrow \mathrm{b} \overline{\mathrm{b}}\right)}{\Gamma\left(\mathrm{Z}^{0} \rightarrow \text { hadrons }\right)} \equiv \frac{\Gamma_{\mathrm{b} \overline{\mathrm{b}}}}{\Gamma_{\mathrm{had}}}
$$

depends on $m_{\mathrm{top}}$, but has negligible uncertainty from the unknown Higgs boson mass and the strong coupling constant $\alpha_{\mathrm{s}}$. A precise measurement of $\Gamma_{\mathrm{b}} / \Gamma_{\text {had }}$ therefore provides a good constraint on the Standard Model parameters. Note that the small number of $b \bar{b}$ pairs produced in the hadronisation process, rather than directly from $\mathrm{Z}^{0}$ decay, are not included in the definition of $\Gamma_{\mathrm{b}} \overline{\mathrm{b}}$.

The fraction $\Gamma_{\mathrm{b}} / \Gamma_{\text {had }}$ is measured by selecting $\mathrm{b} \overline{\mathrm{b}}$ events in hadronic decays of the $\mathrm{Z}^{0}$ using various tagging methods. For the measurement presented here, two different tagging methods are employed: one is to detect electrons or muons coming from semileptonic decays of bottom hadrons, and the other is to find decay vertices of bottom hadrons separated significantly from the primary interaction point. In order to achieve an improved systematic error, the efficiency of the tagging methods is obtained from the data using the double tagging technique. This technique makes use of the fact that each $\mathrm{b} \overline{\mathrm{b}}$ event contains two bottom hadrons produced mostly back-to-back and decaying independently. By applying the tagging methods separately to the two thrust hemispheres in each event, the efficiency can be calculated from the number of tagged hemispheres and the number of events with both hemispheres tagged.

The principle of the double tagging technique is described in the next section. The most important features of the OPAL detector relevant to the analysis are described in section 3 . Section 4 reviews the event samples used, both from real collisions and from simulation. The identification of electrons and muons and the estimation of lepton background are discussed in section 5 . The detection of secondary vertices and a technique to reduce the systematic error due to the detector resolution are described in section 6 . The effect of the tagging efficiency correlation, particularly important for measurements using the double tagging technique, is discussed in section 7 . Section 8 presents the result of the $\Gamma_{\mathrm{b}} / \Gamma_{\text {had }}$ measurement.

\section{Analysis Method}

Each hadronic $\mathrm{Z}^{0}$ decay event is divided into two hemispheres by the plane perpendicular to the thrust axis. The hemispheres are examined separately with two b-tagging methods, lepton tagging and vertex tagging, which are described in the following sections. A hemisphere is said to be tagged if either of the tagging techniques selects it as a b-candidate. The number of tagged hemispheres, $N_{\mathrm{t}}$, and the number of events with two tagged hemispheres, $N_{\mathrm{tt}}$, are counted in a sample of $N_{\text {had }}$ hadronic events. Assuming no correlation between the tagging efficiencies of the two hemispheres in a given event (apart from the underlying flavour dependence), and assuming equal tagging probabilities for $u \bar{u}, d \bar{d}$ and $s \bar{s}$ events, the numbers $N_{\mathrm{t}}$ and $N_{\mathrm{tt}}$ can be expressed as

$$
\begin{aligned}
& N_{\mathrm{t}}=2 N_{\mathrm{had}}\left\{\varepsilon^{\mathrm{b}} \frac{\Gamma_{\mathrm{b} \overline{\mathrm{b}}}}{\Gamma_{\mathrm{had}}}+\varepsilon^{\mathrm{c}} \frac{\Gamma_{\mathrm{c} \overline{\mathrm{c}}}}{\Gamma_{\mathrm{had}}}+\varepsilon^{\mathrm{uds}} \frac{\Gamma_{\mathrm{u} \overline{\mathrm{u}}}+\Gamma_{\mathrm{d} \overline{\mathrm{d}}}+\Gamma_{\mathrm{s} \overline{\mathrm{s}}}}{\Gamma_{\mathrm{had}}}\right\}, \\
& N_{\mathrm{tt}}=N_{\mathrm{had}}\left\{\left(\varepsilon^{\mathrm{b}}\right)^{2} \frac{\Gamma_{\mathrm{b} \overline{\mathrm{b}}}}{\Gamma_{\mathrm{had}}}+\left(\varepsilon^{\mathrm{c}}\right)^{2} \frac{\Gamma_{\mathrm{c} \overline{\mathrm{c}}}}{\Gamma_{\mathrm{had}}}+\left(\varepsilon^{\mathrm{uds}}\right)^{2} \frac{\Gamma_{\mathrm{u} \overline{\mathrm{u}}}+\Gamma_{\mathrm{d} \overline{\mathrm{d}}}+\Gamma_{\mathrm{s} \overline{\mathrm{s}}}}{\Gamma_{\mathrm{had}}}\right\},
\end{aligned}
$$

\footnotetext{
${ }^{1}$ Throughout this paper, the word 'light quark' designates $\mathrm{u}, \mathrm{d}, \mathrm{s}$ and $\mathrm{c}$ quarks. All references to specific particle types are intended also to denote the corresponding antiparticles.
} 
where $\varepsilon^{\mathrm{b}}, \varepsilon^{\mathrm{c}}$ and $\varepsilon^{\mathrm{uds}}$ are the tagging efficiencies for hemispheres in $\mathrm{b} \overline{\mathrm{b}}, \mathrm{c} \overline{\mathrm{c}}$ and other flavours of events, respectively.

The tagging methods are designed to ensure that the efficiency $\varepsilon^{\mathrm{b}}$ for $\mathrm{b} \overline{\mathrm{b}}$ events is much larger than the efficiencies $\varepsilon^{c}$ and $\varepsilon^{\text {uds }}$ for light quark events. Neglecting the contribution from light quark events, the fractional width $\Gamma_{\mathrm{b}} / \Gamma_{\text {had }}$ and the efficiency $\varepsilon^{\mathrm{b}}$ are obtained approximately by

$$
\begin{aligned}
\frac{\Gamma_{\mathrm{b} \bar{b}}}{\Gamma_{\mathrm{had}}} & \approx \frac{N_{\mathrm{t}}^{2}}{4 N_{\mathrm{tt}} N_{\mathrm{had}}}, \\
\varepsilon^{\mathrm{b}} & \approx \frac{2 N_{\mathrm{tt}}}{N_{\mathrm{t}}} .
\end{aligned}
$$

Whereas $\varepsilon^{\mathrm{b}}$ can be obtained from the data themselves, the tagging probabilities $\varepsilon^{\mathrm{c}}$ and $\varepsilon^{\text {uds }}$ have to be estimated with Monte Carlo simulation, which introduces systematic uncertainties. Since the contribution from light quarks gives only a small correction to the measurement, the resulting systematic error is small compared with the single tagging method, which requires good knowledge of the bottom quark efficiency $\varepsilon^{\mathrm{b}}$.

The fractional width for the $c \bar{c}$ final state is predicted by the Standard Model to be $\Gamma_{\mathrm{c} \bar{c}} / \Gamma_{\mathrm{had}}=$ $0.171 \pm 0.001$ for top quark and Higgs particle masses in the ranges $90-250 \mathrm{GeV} / c^{2}$ and $60-1000 \mathrm{GeV} / c^{2}$ respectively [2]. Current measurements of $\Gamma_{\mathrm{c} \overline{\mathrm{c}}} / \Gamma_{\mathrm{had}}$ at LEP are consistent with the Standard Model, and have a combined precision of $\pm 8 \%$ [3]. The fractional width for the three lightest quarks is given by

$$
\frac{\Gamma_{\mathrm{u} \overline{\mathrm{u}}}+\Gamma_{\mathrm{d} \overline{\mathrm{d}}}+\Gamma_{\mathrm{s} \overline{\mathrm{s}}}}{\Gamma_{\mathrm{had}}}=1-\frac{\Gamma_{\mathrm{b} \overline{\mathrm{b}}}}{\Gamma_{\mathrm{had}}}-\frac{\Gamma_{\mathrm{c} \overline{\mathrm{c}}}}{\Gamma_{\mathrm{had}}} .
$$

Once the light-quark tagging probabilities and the $c \overline{\mathbf{c}}$ fractional width are given, equations (1) and (2) can be solved for the two remaining variables: $\Gamma_{\mathrm{b}} / \Gamma_{\text {had }}$ and $\varepsilon^{\mathrm{b}}$.

It should be emphasised that equations (1) and (2) require that the tagging efficiencies of the two hemispheres in an event are correlated only through the flavour of the primary quark pair. This assumption is not strictly valid as there is a small efficiency correlation between hemispheres for physical and instrumental reasons. The effect of this efficiency correlation can, however, be included as a small correction to the result obtained from equations (1) and (2), and will be discussed in section 7 .

\section{The OPAL Detector}

The OPAL detector has been described in reference [4], and only the components important for this analysis are reviewed here. The OPAL coordinate system is a right-handed orthonormal system with its origin at the geometrical centre of the detector. The positive $z$ axis lies along the electron beam direction and $\theta$ and $\phi$ are the polar and azimuthal angles. The $x$ direction points towards the centre of the LEP ring and the $y$ direction points upwards.

The central tracking detectors consist of a silicon microvertex detector, a precision vertex drift chamber, a large volume jet chamber, and thin surrounding z-chambers. The silicon microvertex detector $[5,6]$ has two layers of silicon microstrip detectors, one at a radius of $6.1 \mathrm{~cm}$ with an angular coverage of $|\cos \theta|<0.83$ and one at a radius of $7.5 \mathrm{~cm}$ with an angular coverage of $|\cos \theta|<0.77$. The microvertex detector can provide two measurements of the $\phi$ position for each track with an effective positional resolution of about $10 \mu \mathrm{m}$. The silicon microvertex detector was upgraded [6] before the 1993 data-taking to provide in addition up to two measurements of the $z$ position of each track, but only the $\phi$ information was used for this analysis. When combined with angle and curvature information provided by the other central detector components, the $r$ - $\phi$ impact parameter resolution for $\mathrm{Z}^{0} \rightarrow \mu^{+} \mu^{-}$ 
and $\mathrm{Z}^{0} \rightarrow \mathrm{e}^{+} \mathrm{e}^{-}$events is $18 \mu \mathrm{m}$. The vertex detector is a high resolution drift chamber with axial and stereo wires. The jet chamber, approximately $4 \mathrm{~m}$ long and $3.7 \mathrm{~m}$ in diameter, provides up to 159 space points per track, and measures the ionization energy loss of charged particles [7]. The $z$ coordinates of jet chamber hits are determined using charge division. The precision of the determination of track polar angles is improved by the z-chambers, which provide up to six measurements of the $z$ coordinate on each track. The whole central tracking detector is surrounded by a solenoidal coil which provides a uniform magnetic field of $0.435 \mathrm{~T}$. For the combined central detector, the resolution $\sigma\left(p_{x y}\right)$ of the momentum in the bending plane of the magnetic field is given by $\sigma\left(p_{x y}\right) / p_{x y}=\sqrt{(0.02)^{2}+\left(0.0015 p_{x y}\right)^{2}}$ for $p_{x y}$ in $\mathrm{GeV} / c$. The average resolution of the azimuthal track angle is $0.25 \mathrm{mrad}$. The polar angle resolution varies from $2 \mathrm{mrad}$ for tracks with $\mathrm{z}$-chamber hits to $20 \mathrm{mrad}$ for tracks without them. In hadronic events, the ionization energy loss measurement has a resolution of $3.5 \%$ for tracks with 159 samples.

A lead-glass electromagnetic calorimeter surrounds the magnet coil. The calorimeter is divided into a cylindrical barrel, covering the polar angle range $|\cos \theta|<0.82$, and annular endcaps, covering the range $0.81<|\cos \theta|<0.98$. The barrel calorimeter consists of 9440 lead-glass blocks arranged in a nearly projective geometry. The energy resolution $\sigma_{E}$ of the barrel electromagnetic calorimeter is approximately $\sigma_{E} / E \simeq 2.3 \%$ for beam-momentum electrons from $\mathrm{e}^{+} \mathrm{e}^{-} \rightarrow \mathrm{e}^{+} \mathrm{e}^{-}$events. The resolution on the ratio of the energy to momentum for electrons with energies between 2 and $3 \mathrm{GeV}$ has been measured to be $\sigma(E / p) \approx 10.5 \%$ using $\mathrm{e}^{+} \mathrm{e}^{-} \rightarrow \mathrm{e}^{+} \mathrm{e}^{-} \gamma$ events.

Outside the electromagnetic calorimeter lies the iron return yoke of the magnet, instrumented with streamer tubes as a hadron calorimeter. The muon detectors are placed outside the hadron calorimeter. In total, at least 7 , and in most regions 8 , absorption lengths of material lie between the interaction point and the muon detectors. Muons with momenta above $3 \mathrm{GeV} / c$ usually penetrate to the muon detectors. The muon chambers are constructed as two different detector subsystems in the barrel and endcap parts of the detector. The muon barrel detector covers the polar angle range $|\cos \theta|<0.7$. It has a cylindrical geometry, composed of four layers of planar chambers staggered to resolve left-right ambiguities. The chambers provide coordinate measurements with an accuracy of $1.5 \mathrm{~mm}$ in $r$ - $\phi$, and $2 \mathrm{~mm}$ in $z$. The muon endcap detector covers the polar angle range $0.67<|\cos \theta|<0.98$. It consists of two separated planes of limited streamer tube arrays at each end of the OPAL detector. Resolutions of $1-3 \mathrm{~mm}$ are obtained on the $x$ and $y$ coordinates of hits using the sharing of charge between strips, and the $z$ coordinate is obtained from the surveyed positions of the chambers.

\section{Data Sample and Monte Carlo Simulation}

The data analysed were collected in the 1992 and 1993 data-taking runs of LEP, with centre-of-mass energy at and around the peak of the $\mathrm{Z}^{0}$ resonance. Hadronic $\mathrm{Z}^{0}$ events were selected using an algorithm described in reference [8], additionally requiring that there be at least 7 charged tracks in each event. The extra track multiplicity requirement is predicted to remove most of the small residual background, particularly $\mathrm{Z}^{0}$ decays to tau pairs, remaining in the standard hadronic event selection. Tracks were counted only if they were reconstructed using at least 20 jet chamber hits, had a measured momentum component in the $x-y$ plane of at least $0.15 \mathrm{GeV} / c$, a total measured momentum of less than $65 \mathrm{GeV} / c$, a distance of closest approach to the beam axis of less than $5 \mathrm{~cm}$, and satisfied other minor quality cuts. The hadronic $Z^{0}$ event selection efficiency of these requirements is $(98.1 \pm 0.5) \%$, with a background of less than $0.1 \%$.

Clusters in the barrel (endcap) electromagnetic calorimeter were associated to a charged track if the track pointed to the cluster centroid within $150 \mathrm{mrad}(50 \mathrm{mrad})$ in $\theta$ and $80 \mathrm{mrad}(50 \mathrm{mrad})$ in $\phi$. Each cluster in the barrel (endcap) electromagnetic calorimeter was required to have an energy, corrected for loss in the material in front of the calorimeter, of at least $100 \mathrm{MeV}(200 \mathrm{MeV})$. The thrust 
value and the direction of the thrust axis of each hadronic event were calculated using charged tracks together with electromagnetic clusters with no associated tracks. Two additional cuts were applied to each event; the thrust value, $T$, must be at least 0.8 , and the polar angle of the thrust axis, $\theta_{\text {thrust }}$, must satisfy $\left|\cos \theta_{\text {thrust }}\right|<0.7$. These cuts were designed to ensure good definition of the thrust direction, and to match the acceptance of the silicon microvertex detector.

A total of 746839 hadronic events passed the event selection. Simulation indicated that no significant flavour biases were introduced by any of these selection requirements. The uncertainty due to Monte Carlo statistics, $\pm 0.05 \%$ in $\Gamma_{\mathrm{b}} / \Gamma_{\mathrm{h} a d}$, was taken as the possible systematic error. The effect of an up to $0.1 \%$ background in the hadronic event selection is also included in the systematic error, assuming that this background has a negligible probability of being tagged. The total systematic error due to the event selection is $\pm 0.11 \%$ of the measurement of $\Gamma_{\mathrm{b} \bar{b}} / \Gamma_{\text {had }}$.

Charged tracks and electromagnetic calorimeter clusters with no associated track were combined into jets using the JADE algorithm [9] with the E0 recombination scheme [10]. The invariant masssquared cut-off was set to $x_{\min }=49\left(\mathrm{GeV} / c^{2}\right)^{2}$. The transverse momentum, $p_{t}$, of each track was defined relative to the axis of the jet containing it, where the jet axis was calculated including the momentum of the track.

Monte Carlo simulated events were used for evaluating backgrounds, acceptances for light quark events and efficiency correlations between the two hemispheres of an event. Hadronic events were simulated with the JETSET 7.3 Monte Carlo [11] in conjunction with a program that modelled the response of the OPAL detector [12]. Two samples of simulated events were generated corresponding to the detector configurations in 1992 and 1993 respectively. All simulated events were generated with a centre-of-mass energy of $91.175 \mathrm{GeV}$.

The parameters used in the JETSET program were optimised by OPAL [13]. The fragmentation function of Peterson et al. [14] was used to describe the fragmentation of $b$ and $c$ quarks, with the parameter values $\epsilon_{\mathrm{b}}=0.0055$ and $\epsilon_{\mathrm{c}}=0.05$. Systematic errors due to uncertainties in $\mathrm{b}$ and $\mathrm{c}$ quark fragmentation were evaluated by varying these parameters within the ranges $\epsilon_{\mathrm{b}}=0.0055_{-0.0030}^{+0.0040}$ and $\epsilon_{\mathrm{c}}=0.05 \pm 0.02$, by giving an appropriate weight to each event. These values correspond to average scaled energies of bottom and charm hadrons of $\left\langle x_{E}\right\rangle_{\mathrm{b}}=0.70 \pm 0.02$ and $\left\langle x_{E}\right\rangle_{\mathrm{c}}=0.51 \pm 0.02$ respectively, as measured at LEP [15]. For $\mathrm{u}, \mathrm{d}$ and $\mathrm{s}$ quarks, the Lund symmetric fragmentation function was used.

For semileptonic decays of charmed hadrons, an average branching fraction $B(c \rightarrow \ell)$ of $(9.8 \pm$ $0.5) \%$ was used. This value was obtained by taking the average of the measurements at centreof-mass energies between 9.5 and $39 \mathrm{GeV}$ [16]. The momentum spectra of the leptons in the rest frame of the decaying charmed hadrons were modified according to the refined free-quark model of Altarelli et al. [17]. The two parameters of the model, $m_{\mathrm{s}}$ and $p_{\mathrm{F}}$, were chosen to be $0.001 \mathrm{GeV} / c^{2}$ and $0.467 \mathrm{GeV} / c$ respectively, as given by a fit to DELCO [18] and MARK III [19] data performed by the LEP electroweak heavy flavour working group. Two sets of alternative values of the parameters, $m_{\mathrm{s}}=0.001 \mathrm{GeV} / c^{2}, p_{\mathrm{F}}=0.353 \mathrm{GeV} / c$ and $m_{\mathrm{s}}=0.153 \mathrm{GeV} / c^{2}, p_{\mathrm{F}}=0.467 \mathrm{GeV} / c$, corresponding to the variation allowed by the fit, were used to estimate the systematic error.

The mixture of weakly decaying charmed hadrons $\left(\mathrm{D}^{0}, \mathrm{D}^{+}, \mathrm{D}_{\mathrm{s}}^{+}\right.$and $\left.\Lambda_{\mathrm{c}}^{+}\right)$produced in decays of the $\mathrm{Z}^{0}$ can affect the tagging probability for $\mathbf{c} \overline{\mathbf{c}}$ events because of the large differences in their lifetimes. The fractions of $\mathrm{D}^{0}$ and $\mathrm{D}^{+}$mesons are estimated to be $f\left(\mathrm{D}^{0}\right)=0.557 \pm 0.053 \pm 0.045$ and $f\left(\mathrm{D}^{+}\right)=0.248 \pm 0.037 \pm \mathbf{0 . 0 2 1}$ using the measured production cross-sections times branching ratios at $\sqrt{s}=10.55 \mathrm{GeV}[20]$ and the measured $\mathrm{D}^{0}$ and $\mathrm{D}^{+}$branching fractions [21]. The first errors come from the measurement of individual cross-sections and branching ratios, and the second are the common errors coming from the $\bar{c} \overline{\mathbf{c}}$ cross-section at $\sqrt{s}=10.55 \mathrm{GeV}$. The JETSET Monte Carlo predicts the fractions to be $f\left(\mathrm{D}^{0}\right): f\left(\mathrm{D}^{+}\right): f\left(\mathrm{D}_{\mathrm{s}}^{+}\right): f\left(\Lambda_{\mathrm{c}}^{+}\right)=0.546: 0.257: 0.119: 0.078$, in good agreement with the measured values. In this analysis, production fractions $f\left(\mathrm{D}^{0}\right): f\left(\mathrm{D}^{+}\right): f\left(\mathrm{D}_{\mathrm{s}}^{+}\right): f\left(\Lambda_{\mathrm{c}}^{+}\right)=$ 
$0.557: 0.248: 0.120: 0.075$ were used as the central values. To evaluate the systematic error due to uncertainties in these fractions, the fractions of $\mathrm{D}^{0}$ and $\mathrm{D}^{+}$mesons were varied by the individual and common measurement errors given above while keeping the ratio $f\left(\mathrm{D}_{\mathrm{s}}^{+}\right): f\left(\Lambda_{\mathrm{c}}^{+}\right)$fixed. In addition, the fractions of $\mathrm{D}_{\mathrm{s}}^{+}$mesons and $\Lambda_{\mathrm{c}}^{+}$baryons were varied by \pm 0.050 and $\mp 0.050$ simultaneously, keeping the fractions of other mesons unchanged. The uncertainty on the $\mathrm{D}^{+}$fraction dominates the charmed hadron fraction error on the measured $\Gamma_{\mathrm{b}} / \Gamma_{\mathrm{had}}$. The tagging probability for $c \overline{\mathbf{c}}$ events by leptons is not affected by these charmed hadron fraction uncertainties, because the semileptonic branching ratio $B(c \rightarrow \ell)$ used in this measurement was measured at energies where the mixture of charmed hadrons is expected to be the same as at the $Z^{0}$ resonance.

The lifetimes of the charmed hadrons were varied individually within the errors given by the Particle Data Group [21]. The average charged decay multiplicity for charmed hadrons, which is important for the secondary vertex reconstruction efficiency, has been measured by the MARK III experiment [22] for $\mathrm{D}^{+}, \mathrm{D}^{0}$ and $\mathrm{D}_{\mathrm{s}}^{+}$. Charged tracks from decays of $\mathrm{K}_{\mathrm{S}}^{0}$ and other mesons produced in the charmed hadron decays were included in the measurement. Combining the measured multiplicities using the production fractions given above results in an average of $2.53 \pm 0.08$ charged particles per charmed hadron. A larger uncertainty of \pm 0.5 on the unmeasured charged multiplicity of $\Lambda_{c}^{+}$decays has been included in this error. With the vertex finding algorithm used for this measurement, tracks from $K_{S}^{0}$ decays are rarely included in the secondary vertex, and so the average charged multiplicity excluding $\mathrm{K}_{\mathrm{S}}^{0}$ decay products is relevant to this analysis. The contribution of $\mathrm{K}_{\mathrm{S}}^{0}$ decays is estimated to be 0.31 charged particles per charmed hadron using the measured branching fractions $B\left(\mathrm{D} \rightarrow \mathrm{K}^{0}\right)$ and $B\left(\mathrm{~K}_{\mathrm{S}}^{0} \rightarrow \pi^{+} \pi^{-}\right)$[21]. An average charged multiplicity of 2.22 was therefore used in this measurement. The uncertainty of \pm 0.08 on the charged multiplicity including $K_{S}^{0}$ decays, and the measurement error on the average branching fraction $B\left(\mathrm{D} \rightarrow \mathrm{K}^{0}\right)$ were considered as independent systematic errors.

The production rates of $\mathrm{b}$ and $\mathrm{c}$ quarks in $u \bar{u}, d \overline{\mathrm{d}}$ and $s \overline{\mathrm{s}}$ events via gluon splitting have been calculated within perturbative QCD [23]. The calculation is exact to leading order in $\alpha_{\mathrm{s}}$ and resummed over all orders of leading and next-to-leading logarithmic terms. The calculated rates are expected to be accurate to within $25-30 \%$, and agree with the JETSET prediction to within $30 \%$. The effect of these processes was assessed by removing such events from the Monte Carlo sample, and $50 \%$ of the observed change was taken as the systematic uncertainty.

The systematic error due to uncertainties in $\mathrm{K}^{0}$ and hyperon production rates was assessed by allowing them to vary by $\pm 7.1 \%$ and $\pm 20 \%$ respectively. This corresponds to the precision of OPAL measurements of $\mathrm{K}^{0}$ and hyperon production cross sections and to the accuracy of the modelling by JETSET of their yields [24,25].

In addition, corrections were applied to the simulated events so that they adequately predict the lepton and vertex tagging efficiencies in the real data. These corrections are explained in the next two sections.

\section{Lepton Tagging}

Leptons with high momentum and a large momentum component transverse to the jet direction are a well established signature for $b$ quarks. A large fraction of such leptons are expected to come from semileptonic decays of $b$ hadrons, because of the high mass and the hard fragmentation of the $b$ quark.

Electrons and muons were identified using the algorithms described in references [26] and [27] respectively. Precise knowledge of the identification efficiencies are less important in this analysis, but good estimates of backgrounds, both from misidentified particles and from leptons produced in the decays of non-b hadrons, are essential. 


\subsection{Electron Identification}

Electron candidates were selected in the barrel region of the detector, $|\cos \theta|<0.715$. The momentum, $p$, and the transverse momentum, $p_{t}$, measured with respect to the direction of the jet containing the candidate were required to be at least $2 \mathrm{GeV} / c$ and $1.1 \mathrm{GeV} / c$ respectively. The transverse momentum cut was chosen so that the total error of the $\Gamma_{\mathrm{b}} / \Gamma_{\text {had }}$ measurement is minimized. Electrons from decays of bottom hadrons in this kinematical acceptance were selected with an efficiency of about $69 \%$, with an electron purity of about $89 \%$.

Identification of electrons relies on the specific ionization loss, $\mathrm{d} E / \mathrm{d} x$, measured in the jet chamber and on the total absorption of the energy in the electromagnetic calorimeter. The most probable value of the measured $\mathrm{d} E / \mathrm{d} x$ is about $10 \mathrm{keV} / \mathrm{cm}$ for electrons, and is expected to be constant in the momentum region of interest. The number of jet chamber hits used in the $\mathrm{d} E / \mathrm{d} x$ measurement determines the measurement error, and was required to be at least 40 to ensure good background rejection. A variable $E_{\text {cone }}$ is defined for each track as the energy measured in the lead-glass blocks that are included in the electromagnetic cluster associated to the track and have their centres within $30 \mathrm{mrad}$ of the extrapolated track position. The measured energy was corrected for losses in the material in front of the calorimeter. At least three measurements from the $\mathrm{z}$-chambers were required to improve polar-angle matching between the track and the electromagnetic cluster. The ratio $E_{\text {cone }} / p$ is expected to be close to unity for electrons, and smaller for other particles. The measurement error is given by the sum of the contributions from the measurements of $p$ and $E_{\text {cone }}$, and depends on the momentum and the polar angle of the track.

Two variables, $N_{\mathrm{d} E / \mathrm{d} x}^{\sigma}$ and $N_{E_{\text {cone }} / p}^{\sigma}$, are defined as the deviation of the measured values of $\mathrm{d} E / \mathrm{d} x$ and $E_{\text {cone }} / p$ from their expected values for an electron, divided by the measurement errors. Ideally, both variables should have normal distributions with mean values of zero and widths of unity for electrons. The expected values and the measurement errors were determined empirically by fitting the distributions of the relevant variables. The distribution of the variable $N_{E_{\text {cone }} / p}$ for electron candidates is, however, not symmetric about zero because of radiation in the detector material and lateral leakage of energy from the cone, and is defined so that the negative side of the distribution resembles a unitwidth Gaussian as closely as possible. Figure 1 shows typical distributions of $N_{\mathrm{d} E / \mathrm{d} x}^{\sigma}$ and $N_{E_{\text {cone }} / p}$ for tracks in three momentum intervals in hadronic events. Electron candidates were selected with the cuts $N_{\mathrm{d} E / \mathrm{d} x}^{\sigma}>-1.25$ and $N_{E_{\text {cone }} / p}^{\sigma}>-2$.

Electron candidates were rejected if there was an oppositely charged track in the same event that formed a vertex with the electron candidate consistent with that of a photon conversion. The algorithm for recognising photon conversions is described in reference [27]. The efficiency of conversion rejection and the probability of the rejected tracks really being conversion products were estimated to be $(78.1 \pm 5.6) \%$ and $(78.6 \pm 4.0) \%$ respectively.

The conversion rejection removed 851 tracks, leaving a total of 9917 hemispheres containing electron candidates in the data.

Electrons in simulated events were subject to the same identification algorithm used for the data. The expected value and the error on $\mathrm{d} E / \mathrm{d} x$ used to define the variable $N_{\mathrm{d} E / \mathrm{d} x}^{\sigma}$ were determined by the same procedure used for the real data. The resolution on $N_{E_{\text {cone }} / p}$ was corrected by randomly smearing the value of $E_{\text {cone }}$ to reproduce the resolution measured in the data. The smearing decreases the efficiency of the $N_{E_{\text {cone }} / p}^{\sigma}$ cut by $1.8 \%$, to which a $1.1 \%$ systematic error was assigned. The efficiencies for tracks to have a sufficient number of $\mathrm{z}$-chamber hits and $\mathrm{d} E / \mathrm{d} x$ hits were measured for all tracks within the geometrical acceptance, in both data and simulation, and the corrections derived were applied to the simulated sample. After the correction, the efficiency difference between the real and simulated data was studied using several subsamples of tracks in different angular, momentum, and transverse momentum ranges, and using muon candidates identified by the algorithm described 
in the next section. The average electron identification efficiency was modelled by the Monte Carlo simulation within a relative systematic uncertainty of $\pm 2.5 \%$.

The electron identification efficiency depends on the track environment (concentration of particles in the neighbourhood of the track), and thus on the event flavour. For the purpose of the double tagging measurement, the efficiency for electrons in $c \bar{c}$ events is of primary importance, while the comparison between the real data and the Monte Carlo can be done only for the average of all flavours. The efficiency difference between $c \bar{c}$ and all flavours of events as predicted by the Monte Carlo was assigned a $50 \%$ uncertainty for each of the electron identification selection cuts, which led to an additional $3.2 \%$ systematic error. Including this uncertainty, the total relative systematic uncertainty in the electron identification efficiency was taken to be $\pm 4.0 \%$.

\subsection{Muon Identification}

The identification algorithm for muons was unchanged from that of reference [27]. Tracks with polar angles satisfying $|\cos \theta|<0.9$, momenta $p>3 \mathrm{GeV} / c$ and transverse momenta $p_{t}>1.2 \mathrm{GeV} / c$ were considered as muon candidates. The transverse momentum cut was optimized in order to minimize the total measurement error as in the case of electrons. The selection efficiency for muons from bottom hadron decays in this kinematical acceptance was about $76 \%$, with a muon purity of around $88 \%$.

Identification of muons relied on their penetrating nature. Track segments were reconstructed in the four-layer external muon chambers independently of tracks found in the central detectors. The central tracks were extrapolated through the material and magnetic field of the detector to the muon chambers. The presence of a matching segment and the quality of the positional match between the extrapolated track and the muon segment were used to identify muons. The angular separation of the point of closest approach of the extrapolation to the segment was evaluated in $\phi$ and $\theta$. A matching parameter, $\chi_{\text {pos }}$, was constructed by adding these differences in quadrature, first normalizing each by its expected error. The error includes both reconstruction and multiple scattering uncertainties.

Muon candidates were selected by requiring $\chi_{\text {pos }}<3$, considering only the best matched muon segment for each central track, and only the best matched central track for each muon segment. In addition, a small number of pathological cases were removed by requiring that there be not more than 20 muon segments reconstructed within $300 \mathrm{mrad}$ in $\phi$ of the best-matched segment. Finally, the measured $\mathrm{d} E / \mathrm{d} x$ was required to be consistent with a muon, by requiring it to be greater than the value expected for a muon or less than 1.96 standard deviations below the muon expectation. This latter requirement rejected $60 \%$ of charged kaon tracks otherwise misidentified as muons, but removed only $4 \%$ of muons from $\mathrm{b}$ and $\mathrm{c}$ decays. The $\mathrm{d} E / \mathrm{d} x$ requirement was not applied to the small number of tracks which had less than 20 hits used in the $\mathrm{d} E / \mathrm{d} x$ measurement.

A total of 10953 hemispheres containing muon candidates were found in the data.

The efficiency of the muon identification criteria was studied with various control samples of identified particles. Muon pair events from decays of the $Z^{0}$ and from two-photon collisions were used to study the accuracy of the modelling of the efficiency by the Monte Carlo. The effect of nearby track activity on the identification efficiency was studied using various Monte Carlo and data samples, and found to be small. Identified charged pions from $\mathrm{K}^{0}$ decays were used to measure the efficiency of the $\mathrm{d} E / \mathrm{d} x$ cut, and to derive a systematic error due to it.

Overall, small corrections were found to be needed to the predicted Monte Carlo efficiency, of $+1.1 \%$ in the barrel part of the detector, particularly important for this analysis, and of $-1.2 \%$ in the endcap part. The total relative systematic uncertainty on the efficiency as modelled by the Monte Carlo was $1.7 \%$. There was no significant dependence of the muon identification efficiency on the event flavour. 


\subsection{Background Subtraction}

Three types of background to the identified electrons and muons were considered: photon conversions, hadrons misidentified as electrons, and hadrons misidentified as muons.

As explained in section 5.1, most of the electrons arising from photon conversions were identified and rejected. The number of photon conversions left in the sample after the rejection was estimated from the number of rejected candidates, using the rejection efficiency and the probability of correct rejection given in the previous section. This remaining background amounts to $(1.9 \pm 0.7) \%$ of the electron signal and was subtracted from the number of tagged hemispheres.

The amount of hadronic background in the electron candidate sample was estimated from the data using the distributions of $N_{\mathrm{d} E / \mathrm{d} x}^{\sigma}$ and $N_{E_{\text {cone }} / p}^{\sigma}$. The method relies on the assumption that the distributions of the two variables for background tracks are uncorrelated. Since the variables $N_{\mathrm{d} E / \mathrm{d} x}^{\sigma}$ and $N_{E_{\text {cone }} / p}^{\sigma}$ were defined using the expected resolution of $\mathrm{d} E / \mathrm{d} x$ and $E_{\text {cone }} / p$, which depend on the momentum $p$ and the polar angle $|\cos \theta|$ of the track, the distributions of these variables for nonelectron tracks also depend on $p$ and $|\cos \theta|$. To ensure the validity of the above assumption of no correlation, the tracks in the data were divided into small bins of $p$ and $|\cos \theta|$. In each bin, the twodimensional distribution of $N_{\mathrm{d} E / \mathrm{d} x}^{\sigma}$ and $N_{E_{\mathrm{cone}} / p}^{\sigma}$ was made for tracks passing the electron identification criteria except for the cuts on these two variables. The amount of hadronic background in the region selected by the electron identification criteria $\left(N_{\mathrm{d} E / \mathrm{d} x}^{\sigma}>-1.25\right.$ and $\left.N_{E_{\text {cone }} / p}^{\sigma}>-2\right)$ was estimated from the distribution outside the region. The effect of the tail of the distribution of true electrons failing the selection cuts was taken into account. The estimated backgrounds are shown in figure 1 as hatched histograms under the signal. The background in the sample of identified electrons was estimated to be 916.9 candidates. The overall relative systematic uncertainty on this background number was estimated to be $\pm 7.2 \%$.

The background in the identified muon sample comes mainly from four origins: decays-in-flight of pions and kaons, hadronic showers leaking through the absorber material, hadrons penetrating the absorber without interacting strongly, and incorrect association of tracks to muon chamber signals of unrelated origin. The amount of background in the real data was calculated in bins of $p$ and $p_{t}$ by multiplying the fake probability per track, measured in the Monte Carlo, by the number of charged tracks in the data. This procedure avoids relying on the Monte Carlo simulation to predict the $p$ and $p_{t}$ distribution of the tracks. In addition, an overall multiplicative correction factor of 1.13 was applied to the total predicted background, derived by comparing the Monte Carlo prediction of the muon fake rate with several background control samples. These samples included $\mathrm{K}^{0}$ decays to $\pi^{+} \pi^{-}$, three-prong $\tau$ decays, and samples of tracks that failed to pass one or more of the muon identification criteria. The estimated background in the selected muon sample was 1289.8 candidates. The overall relative uncertainty on the muon background level was estimated from these tests to be $\pm 9 \%$.

Lepton backgrounds were subtracted not only from the number of tagged hemispheres, but also from the number of double-tagged events, as follows. For photon conversions and muon backgrounds, the same methods explained above were applied to tracks in hemispheres opposite to tagged hemispheres. For electron candidates, however, statistics did not allow this procedure to give a useful estimate of the background contamination in double-tagged events. The fake probability per track obtained for all events was therefore used instead to estimate the background in the double-tagged events. The fake probability was calculated in bins of $p$ and $p_{t}$ as the number of estimated background tracks divided by the number of tracks failing the electron identification only because of the $N_{\mathrm{d} E / \mathrm{d} x}^{\sigma}$ requirement. The number of tracks similarly failing just the $N_{\mathrm{d} E / \mathrm{d} x}^{\sigma}$ cut in the hemisphere opposite each tagged hemisphere was multiplied by this fake probability, giving the estimate of the background in the double-tagged events. The Monte Carlo simulation predicted a $(5.0 \pm 4.3) \%$ higher fake probability in $\mathrm{b} \overline{\mathrm{b}}$ events than the average of the five flavours, where the error is due to the Monte Carlo statistics. Considering this as a systematic uncertainty, an additional systematic error of $\pm 5.0 \%$ was 
applied to the estimate of the contribution of the electron background to the number of double-tagged events. The probability of an event having two background leptons, one in each hemisphere, was estimated to be about $4 \times 10^{-6}$ and was neglected.

\section{$6 \quad$ Vertex Tagging}

Hadronic $\mathrm{Z}^{0}$ decays into bottom quarks can be enriched by taking advantage of the relatively long $(\sim 1.5 \mathrm{ps})$ lifetimes of bottom hadrons. In this analysis, the detection of secondary vertices significantly separated from the primary vertex was adopted to exploit this long lifetime. As the double tagging technique enables us to derive the tagging efficiency for $b \bar{b}$ events from the data, the measurement does not depend on the knowledge of the bottom hadron lifetimes and decay multiplicities and the $\mathrm{b}$ quark fragmentation, and is also relatively insensitive to the uncertainty in the tracking resolution of the detector. In addition, an extension of the double tagging method, the folded double tagging method, is introduced to reduce further the effect of uncertainty in the charged track resolution.

\subsection{Vertex Reconstruction}

The primary vertex for each event was reconstructed using a $\chi^{2}$ minimisation method which also incorporated the average beam spot position derived from the data as a constraint in the vertex fit. The beam spot position itself was used for events failing the primary vertex finding, amounting to about $0.1 \%$ of the total number of events.

Charged tracks used for secondary vertex reconstruction were required to have a momentum greater than $500 \mathrm{MeV} / c$. In addition, the impact parameter relative to the reconstructed primary vertex was required to satisfy $\left|d_{0}\right|<0.3 \mathrm{~cm}$, and its error $\sigma_{d_{0}}<0.1 \mathrm{~cm}$. This mainly removes poorly measured tracks and, for example, tracks from $\mathrm{K}^{0}$ or $\Lambda^{0}$ decays.

Secondary vertex finding was carried out separately for each reconstructed jet in an event. In a first iteration, all the charged tracks in a given jet were fitted to a common vertex point in the $x-y$ plane. If one or more charged tracks contributed $\Delta \chi^{2}>4$ to the overall $\chi^{2}$ for the secondary vertex fit, then the track with the largest $\Delta \chi^{2}$ was removed and the fit repeated. This process was continued until all tracks contributed $\Delta \chi^{2}<4$ or until fewer than four charged tracks remained, in which case the secondary vertex reconstruction failed for this particular jet.

For each reconstructed secondary vertex, the vertex decay length $L$ was defined as the distance of the secondary vertex from the primary vertex, constrained by the direction given by the total momentum vector (in the plane transverse to the beam direction) of the tracks assigned to the secondary vertex. The total vertex momentum vector was also used to determine the sign of the decay length; $L>0$ if the secondary vertex was displaced from the primary vertex in the same direction as the total momentum, and $L<0$ otherwise.

Each event hemisphere was assigned a vertex tag if it contained at least one secondary vertex with a signed decay length significance (defined as the signed decay length $L$ divided by its error $\sigma_{L}$ ) greater than a given value. The central value for the $L / \sigma_{L}$ cut $\left(L / \sigma_{L}>8\right)$ was chosen in order to minimise the total error on the measurement of $\Gamma_{\mathrm{b}} / \Gamma_{\text {had }}$.

Figure 2 shows the inclusive $L / \sigma_{L}$ distribution for secondary vertices reconstructed in the data and Monte Carlo event samples. Vertices with large positive values of $L / \sigma_{L}$ are dominantly produced by $\mathrm{b} \overline{\mathrm{b}}$ events. The Monte Carlo lies significantly below the data in this region, but this difference can be due to assumptions in the Monte Carlo about the underlying $b$ quark physics, in particular the average bottom hadron lifetime used in the event generation and the average charged multiplicity of 
bottom hadron decays. In any case, differences between data and Monte Carlo in this region do not affect the double tagging analysis since the $\mathrm{b}$ quark tagging efficiency is measured from the data.

Differences between data and Monte Carlo are also seen in the region around $L / \sigma_{L}=0$ and in the backward half $\left(L / \sigma_{L}<0\right)$ of the decay length distribution. These differences are due largely to an over-optimistic simulation of the detector resolution and hit-association probabilities for charged tracks. Additional smearing was therefore applied to the Monte Carlo events in order to improve the agreement between data and Monte Carlo. The smearing method used applied a single multiplicative smearing factor, $\beta$, to the difference between the reconstructed and true track impact parameters and $\phi$ angle measurements. The distributions of $L / \sigma_{L}$ in the Monte Carlo samples smeared using $\beta=1.2$ and 1.4 are shown in figures $2(\mathrm{a})$ and $2(\mathrm{~b})$ respectively. The distribution with $\beta=1.2$ is slightly narrower than that for the real data, while $\beta=1.4$ is slightly broader. The backward half $\left(L / \sigma_{L}<0\right)$ of the distribution is dominated by the effects of detector resolution, and resolution effects in the region of interest, $L / \sigma_{L}>8$, can be studied by examining the corresponding backward region $L / \sigma_{L}<-8$. A central value $\beta=1.4$ was used for this analysis because of the better agreement with the data in this region, while the change in the measured value of $\Gamma_{\mathrm{b} \overline{\mathrm{b}}} / \Gamma_{\mathrm{had}}$ using $\beta=1.2$ and $\beta=1.4$ (i.e., a $20 \%$ variation in detector resolution) was used to estimate the systematic error. More precisely, the larger of either the full difference between $\beta=1.4$ and 1.2 or the statistical error of the difference was taken as the systematic error due to the detector resolution.

\subsection{Folded Double Tagging}

The decay length significance $L / \sigma_{L}$ is an inherently symmetric variable: its distribution should be symmetric about $L / \sigma_{L}=0$ if there are no particles with detectable lifetime. The backward half $\left(L / \sigma_{L}<0\right)$ of the decay length distribution can be used to control the systematic uncertainty of the vertex tagging efficiency coming from the understanding of the detector resolution. In order to do this, not only a forward vertex tag such as $L / \sigma_{L}>8$ was considered, but also the corresponding backward $\operatorname{tag} L / \sigma_{L}<-8$. For light quark events, any change in the detector resolution is expected to increase or decrease the fractions of forward and backward tags by similar amounts, but their difference will be relatively insensitive to such a change.

Five quantities are counted in the data after dividing the events into hemispheres:

- $N_{\mathrm{v}}$ the number of forward tagged hemispheres,

- $N_{\overline{\mathrm{v}}}$ the number of backward tagged hemispheres,

- $N_{\mathrm{vv}}$ the number of events for which both hemispheres receive a forward tag,

- $N_{\overline{\mathrm{vv}}}$ the number of events for which both hemispheres receive a backward tag,

- $N_{\mathrm{v} \overline{\mathrm{v}}}$ the number of events for which one hemisphere receives a forward tag and the other a backward tag.

Assuming no correlations between the forward and backward tagging probabilities for the two hemispheres in a multihadron event, we have:

$$
\begin{aligned}
& N_{\mathrm{v}}=2 N_{\text {had }}\left\{\varepsilon_{\mathrm{v}}^{\mathrm{b}} \frac{\Gamma_{\mathrm{b} \overline{\mathrm{b}}}}{\Gamma_{\mathrm{had}}}+\varepsilon_{\mathrm{v}}^{\mathrm{c}} \frac{\Gamma_{\mathrm{c} \overline{\mathrm{c}}}}{\Gamma_{\mathrm{had}}}+\varepsilon_{\mathrm{v}}^{\mathrm{uds}} \frac{\Gamma_{\mathrm{u} \overline{\mathrm{u}}}+\Gamma_{\mathrm{d} \overline{\mathrm{d}}}+\Gamma_{\mathrm{s} \overline{\mathrm{s}}}}{\Gamma_{\mathrm{had}}}\right\}, \\
& N_{\overline{\mathrm{v}}}=2 N_{\text {had }}\left\{\varepsilon_{\overline{\mathrm{v}}} \frac{\Gamma_{\mathrm{b} \overline{\mathrm{b}}}}{\Gamma_{\mathrm{had}}}+\varepsilon_{\overline{\mathrm{v}}}^{\mathrm{c}} \frac{\Gamma_{\mathrm{c} \overline{\mathrm{c}}}}{\Gamma_{\mathrm{had}}}+\varepsilon_{\overline{\mathrm{v}}}^{\mathrm{uds}} \frac{\Gamma_{\mathrm{u} \overline{\mathrm{u}}}+\Gamma_{\mathrm{d} \overline{\mathrm{d}}}+\Gamma_{\mathrm{s} \overline{\mathrm{s}}}}{\Gamma_{\mathrm{had}}}\right\},
\end{aligned}
$$




$$
\begin{aligned}
& N_{\mathrm{vv}}=N_{\mathrm{had}}\left\{\left(\varepsilon_{\mathrm{v}}^{\mathrm{b}}\right)^{2} \frac{\Gamma_{\mathrm{b} \overline{\mathrm{b}}}}{\Gamma_{\mathrm{had}}}+\left(\varepsilon_{\mathrm{v}}^{\mathrm{c}}\right)^{2} \frac{\Gamma_{\mathrm{c} \overline{\mathrm{c}}}}{\Gamma_{\mathrm{had}}}+\left(\varepsilon_{\mathrm{v}}^{\mathrm{uds}}\right)^{2} \frac{\Gamma_{\mathrm{u} \overline{\mathrm{u}}}+\Gamma_{\mathrm{d} \overline{\mathrm{d}}}+\Gamma_{\mathrm{s} \overline{\mathrm{s}}}}{\Gamma_{\mathrm{had}}}\right\}, \\
& N_{\overline{\mathrm{vv}}}=N_{\mathrm{had}}\left\{\left(\varepsilon_{\overline{\mathrm{v}}}^{\mathrm{b}}\right)^{2} \frac{\Gamma_{\mathrm{b} \overline{\mathrm{b}}}}{\Gamma_{\mathrm{had}}}+\left(\varepsilon_{\overline{\mathrm{v}}}^{\mathrm{c}}\right)^{2} \frac{\Gamma_{\mathrm{c} \overline{\mathrm{c}}}}{\Gamma_{\mathrm{had}}}+\left(\varepsilon_{\overline{\mathrm{v}}}^{\mathrm{uds}}\right)^{2} \frac{\Gamma_{\mathrm{u} \overline{\mathrm{u}}}+\Gamma_{\mathrm{d} \overline{\mathrm{d}}}+\Gamma_{\mathrm{s} \overline{\mathrm{s}}}}{\Gamma_{\mathrm{had}}}\right\}, \\
& N_{\mathrm{v} \overline{\mathrm{v}}}=2 N_{\mathrm{had}}\left\{\varepsilon_{\mathrm{v}}^{\mathrm{b}} \varepsilon_{\overline{\mathrm{v}}} \frac{\Gamma_{\mathrm{b} \overline{\mathrm{b}}}}{\Gamma_{\mathrm{had}}}+\varepsilon_{\mathrm{v}}^{\mathrm{c}} \varepsilon_{\overline{\mathrm{v}}}^{\mathrm{c}} \frac{\Gamma_{\mathrm{c} \overline{\mathrm{c}}}}{\Gamma_{\mathrm{had}}}+\varepsilon_{\mathrm{v}}^{\mathrm{uds}} \varepsilon_{\overline{\mathrm{v}}}^{\mathrm{uds}} \frac{\Gamma_{\mathrm{u} \overline{\mathrm{u}}}+\Gamma_{\mathrm{d} \overline{\mathrm{d}}}+\Gamma_{\mathrm{s} \overline{\mathrm{s}}}}{\Gamma_{\mathrm{had}}}\right\},
\end{aligned}
$$

where $\varepsilon_{\mathrm{v}}^{\mathrm{b}}, \varepsilon_{\mathrm{v}}^{\mathrm{c}}$ and $\varepsilon_{\mathrm{v}}^{\mathrm{uds}}$ are the probabilities of obtaining a forward vertex tag in a hemisphere in $\mathrm{b} \overline{\mathrm{b}}$, $\mathbf{c} \overline{\mathbf{c}}$ and other light quark events respectively, and $\varepsilon_{\overline{\mathrm{v}}}^{\mathrm{b}}, \varepsilon_{\overline{\mathrm{v}}}^{\mathrm{c}}$ and $\varepsilon_{\overline{\mathrm{v}}}^{\text {uds }}$ are the corresponding probabilities for a backward vertex tag. Equations (6) to (10) can be combined to give the folded double tagging equations:

$$
\begin{aligned}
N_{\mathrm{v}}-N_{\overline{\mathrm{v}}} & =2 N_{\mathrm{had}}\left\{\left(\varepsilon_{\mathrm{v}}^{\mathrm{b}}-\varepsilon_{\overline{\mathrm{v}}}^{\mathrm{b}}\right) \frac{\Gamma_{\mathrm{b} \overline{\mathrm{b}}}}{\Gamma_{\mathrm{had}}}+\left(\varepsilon_{\mathrm{v}}^{\mathrm{c}}-\varepsilon_{\overline{\mathrm{v}}}^{\mathrm{c}}\right) \frac{\Gamma_{\mathrm{c} \overline{\mathrm{c}}}}{\Gamma_{\mathrm{had}}}+\left(\varepsilon_{\mathrm{v}}^{\mathrm{uds}}-\varepsilon_{\overline{\mathrm{v}}}^{\mathrm{uds}}\right) \frac{\Gamma_{\mathrm{u} \overline{\mathrm{u}}}+\Gamma_{\mathrm{d} \overline{\mathrm{d}}}+\Gamma_{\mathrm{s} \overline{\mathrm{s}}}}{\Gamma_{\mathrm{had}}}\right\}, \\
N_{\mathrm{vv}}-N_{\mathrm{v} \overline{\mathrm{v}}}+N_{\overline{\mathrm{v} v}} & =N_{\mathrm{had}}\left\{\left(\varepsilon_{\mathrm{v}}^{\mathrm{b}}-\varepsilon_{\overline{\mathrm{v}}}^{\mathrm{b}}\right)^{2} \frac{\Gamma_{\mathrm{b} \overline{\mathrm{b}}}}{\Gamma_{\mathrm{had}}}+\left(\varepsilon_{\mathrm{v}}^{\mathrm{c}}-\varepsilon_{\overline{\mathrm{v}}}^{\mathrm{c}}\right)^{2} \frac{\Gamma_{\mathrm{c} \overline{\mathrm{c}}}}{\Gamma_{\mathrm{had}}}+\left(\varepsilon_{\mathrm{v}}^{\mathrm{uds}}-\varepsilon_{\overline{\mathrm{v}}}^{\mathrm{uds}}\right)^{2} \frac{\Gamma_{\mathrm{u} \overline{\mathrm{u}}}+\Gamma_{\mathrm{d} \overline{\mathrm{d}}}+\Gamma_{\mathrm{s} \overline{\mathrm{s}}}}{\Gamma_{\mathrm{had}}}\right\}
\end{aligned}
$$

These equations have the same form as equations (1) and (2) with the substitutions:

$$
\begin{aligned}
N_{\mathrm{t}} & \rightarrow N_{\mathrm{v}}-N_{\overline{\mathrm{v}}}, \\
N_{\mathrm{tt}} & \rightarrow N_{\mathrm{vv}}-N_{\mathrm{v} \overline{\mathrm{v}}}+N_{\overline{\mathrm{vv}}}, \\
\varepsilon^{\mathrm{b}} & \rightarrow \varepsilon_{\mathrm{v}}^{\mathrm{b}}-\varepsilon_{\overline{\mathrm{v}}}^{\mathrm{b}}, \\
\varepsilon^{\mathrm{c}} & \rightarrow \varepsilon_{\mathrm{v}}^{\mathrm{c}}-\varepsilon_{\overline{\mathrm{v}}}^{\mathrm{c}}, \\
\varepsilon^{\mathrm{uds}} & \rightarrow \varepsilon_{\mathrm{v}}^{\mathrm{uds}}-\varepsilon_{\overline{\mathrm{v}}}^{\mathrm{uds}} .
\end{aligned}
$$

The folded double tagging measurement can then be carried out following a similar procedure to that given in section 2, except that now equations (11) and (12) are solved for the two unknowns $\left(\varepsilon_{\mathrm{v}}^{\mathrm{b}}-\varepsilon_{\overline{\mathrm{v}}}^{\mathrm{b}}\right)$ and $\Gamma_{\mathrm{b}} / \Gamma_{\mathrm{had}}$. In place of the light flavour tagging efficiencies $\varepsilon^{\mathrm{c}}$ and $\varepsilon^{\text {uds }}$, the differences of the forward and backward tagging probabilities $\left(\varepsilon_{\mathrm{v}}^{\mathrm{c}}-\varepsilon_{\overline{\mathrm{v}}}^{\mathrm{c}}\right)$ and $\left(\varepsilon_{\mathrm{v}}^{\text {uds }}-\varepsilon_{\overline{\mathrm{v}}}^{\text {uds }}\right)$ need to be estimated from Monte Carlo simulation. This results in a measurement more robust against the uncertainty in the detector resolution. We note that, in practice, the backward efficiency for $\mathrm{b} \overline{\mathrm{b}}$ events, $\varepsilon_{\overline{\mathrm{v}}}^{\mathrm{b}}$, is much smaller than the forward efficiency $\varepsilon_{\mathrm{v}}^{\mathrm{b}}$ so that $\left(\varepsilon_{\mathrm{v}}^{\mathrm{b}}-\varepsilon_{\overline{\mathrm{v}}}^{\mathrm{b}}\right)$ is nearly equal to $\varepsilon_{\mathrm{v}}^{\mathrm{b}}$.

\section{Efficiency Correlation}

As mentioned in section 2, the double-tagging method used in this measurement relies on the assumption that the probabilities of the two hemispheres of an event being tagged are correlated only through the flavour of the initial quark pair. This assumption, expressed inherently in equations (1) and (2), is not perfectly correct for three reasons: the existence of kinematical correlations due to final state radiation, of geometrical correlations due to detector non-uniformities, and of correlations coming from the determination of the primary vertex. The effect of the tagging efficiency correlation for $b \bar{b}$ events can be introduced into equation (2) by replacing the $\left(\varepsilon^{\mathrm{b}}\right)^{2}$ with $C\left(\varepsilon^{\mathrm{b}}\right)^{2}$, where the multiplicative parameter $C$ should be close to unity. The approximate solution given in equation (3) then becomes

$$
\frac{\Gamma_{\mathrm{b} \overline{\mathrm{b}}}}{\Gamma_{\mathrm{had}}} \approx \frac{C N_{\mathrm{t}}^{2}}{4 N_{\mathrm{tt}} N_{\mathrm{had}}},
$$

i.e., the result should be multiplied by $C$ to take the efficiency correlation into account. The different contributions to $C$ are discussed below. 
A hadronic decay of a $\mathrm{Z}^{0}$ may have one or more gluons carrying a substantial amount of energy. In such an event, there is less energy available for the primary quark pair than in a simple two-jet event, thus resulting in a smaller chance of being tagged by high momentum leptons or by vertices. In addition, the thrust axis may not represent well the directions of the primary quarks due to the presence of the hard gluon jets. The JETSET Monte Carlo predicts that $1.43 \%$ of $\mathrm{b} \overline{\mathrm{b}}$ events passing the event selection have both bottom hadrons in one thrust hemisphere. These events represent $0.89 \%$ of all tagged hemispheres, due to the lower tagging efficiency of such events. As these events do not contribute to the double-tagging event sample, they decrease the double-tagging efficiency by $1.43 \%$ and the single-tagging efficiency by $(1.43-0.89) \%$. The correlation $C$ is given by dividing the doubletagging efficiency by the single-tagging efficiency squared, and is approximately $C=0.9965 \pm 0.0019$, where the error is due to Monte Carlo statistics.

After removing the same-hemisphere events, the Monte Carlo predicted the momenta of the two bottom hadrons still to be correlated with each other. The size of the correlation can be characterized by

$$
\frac{\left\langle p_{\mathrm{B}} \cdot p_{\overline{\mathrm{B}}}\right\rangle}{\left\langle p_{\mathrm{B}}\right\rangle\left\langle p_{\overline{\mathrm{B}}}\right\rangle}=1.0087,
$$

where $p_{\mathrm{B}}$ and $p_{\overline{\mathrm{B}}}$ are the momenta of bottom hadrons containing $\overline{\mathrm{b}}$ and $\mathrm{b}$ quarks respectively. Using the tagging efficiency $\varepsilon^{\mathrm{b}}$ obtained from the Monte Carlo as a function of the bottom hadron momentum, the momentum correlation was translated to an efficiency correlation as

$$
C=\frac{\left\langle\varepsilon^{\mathrm{b}}\left(p_{\mathrm{B}}\right) \varepsilon^{\mathrm{b}}\left(p_{\overline{\mathrm{B}}}\right)\right\rangle}{\left\langle\varepsilon^{\mathrm{b}}\left(p_{\mathrm{B}}\right)\right\rangle\left\langle\varepsilon^{\mathrm{b}}\left(p_{\overline{\mathrm{B}}}\right)\right\rangle}=1.0043 \pm \mathbf{0 . 0 0 0 2},
$$

where the error is due to Monte Carlo statistics. Small inaccuracies in the modelling of the tagging efficiency are expected to have little effect on this estimate, because only the momentum dependence is relevant.

Combining the effects of same-hemisphere events and the bottom hadron momentum correlation, the overall correlation caused by gluon emission was estimated to be $C=1.0008 \pm 0.0019$ where the error is due to Monte Carlo statistics. The systematic uncertainty on the correlation measurement was studied by generating Monte Carlo samples with different values of the b-quark fragmentation parameter $\epsilon_{\mathrm{b}}$ and the $\Lambda_{\mathrm{QCD}}$ parameter, and using the JETSET matrix element event generator in place of the parton shower generator. The largest difference, $-\mathbf{0 . 0 0 2 4}$, was observed when the matrix element event generator was used, and was taken as the systematic error. The effects of the other parameters were found to be negligible.

The two bottom hadrons in a $\mathrm{b} \overline{\mathrm{b}}$ event are likely to be produced back-to-back. Their decay products are therefore likely to strike geometrically opposite parts of the detector. This introduces an efficiency correlation if the efficiency of the detector is not completely uniform. This correlation can be estimated by measuring the hemisphere tagging probability in the real data as a function of the thrust axis direction as

$$
C=\frac{4\left\langle f^{+}(\theta, \phi) f^{-}(\theta, \phi)\right\rangle}{\left\langle f^{+}(\theta, \phi)+f^{-}(\theta, \phi)\right\rangle^{2}},
$$

where $f^{+}$and $f^{-}$are the fraction of tagged hemispheres in the $+z$ and $-z$ directions respectively, and the average is taken over the full solid angle acceptance. The actual estimation was carried out in small bins of $|\cos \theta|$ and $\phi$ and the effect of statistical fluctuation of the measurement of $f$ was assessed by a Monte Carlo technique. The correlation $C$ was estimated to be $1.0051 \pm 0.0009$, where the error is statistical.

The vertex tag requires knowledge of the primary vertex position, which is shared by both hemispheres. Since the primary vertex was determined event by event, based on the fit of the tracks in the whole event, this can also cause an efficiency correlation between the hemispheres. The effect of this 
correlation was studied by analysing the Monte Carlo simulated data using the true primary vertex position in place of the measured one. The observed change in the measured value of $\Gamma_{\mathrm{b}} / \Gamma_{\text {had }}$ using only vertex tags was $+\mathbf{0 . 0 9 \%}$. The corresponding change in the combined result, $+0.06 \%$, was taken as the systematic error.

Overall, the efficiency correlation parameter $C$ for $b \bar{b}$ events was estimated to be $1.0059 \pm 0.0032$. A similar efficiency correlation is also expected for light quark events. Such a correlation has a negligible effect on the measurement, however.

\section{Result}

The numbers of tagged hemispheres and double-tagged events found in the 746839 events of hadronic $\mathrm{Z}^{0}$ decays are listed in table 1 . The data samples collected in 1992 and 1993 were analysed independently because the upgrade of the silicon microvertex detector was expected to result in slightly different tagging efficiencies. The symbols $N_{i}$ denote the numbers of hemispheres tagged by $i$, where

$$
i= \begin{cases}\ell & \text { for either electrons or muons, } \\ \mathrm{v} & \text { for forward vertices, } \\ \overline{\mathrm{v}} & \text { for backward vertices, } \\ \mathrm{a} & \text { for either leptons or forward vertices. }\end{cases}
$$

The symbols $N_{i j}$ denote the numbers of double-tagged events with one hemisphere tagged by $i$ and the other by $j$. The backgrounds to the identified leptons, discussed in section 5.3, were subtracted from the totals before solving for $\Gamma_{\mathrm{b}} / \Gamma_{\text {had }}$ and $\varepsilon^{\mathrm{b}}$ because they were determined inclusively for the lepton-tagged samples. Incorrectly reconstructed vertices, on the other hand, are instead included in $\varepsilon^{c}$ and $\varepsilon^{\text {uds }}$, since they are estimated from Monte Carlo separately for the different event flavours.

The hemisphere tagging probabilities for light quark events, estimated using Monte Carlo events, are given in per cent in table 2. In combining the lepton and vertex tags, each tagged hemisphere was counted only once even if it was tagged by more than one type of tag. In principle, the tagging efficiencies for light quark events were therefore slightly smaller than the sum of the lepton and vertextagging efficiencies for those events. This affected the final result by less than $0.1 \%$, however, and was neglected. It is irrelevant for $\mathrm{b}$ quark events, since the tagging efficiency was determined directly from the data.

\begin{tabular}{ll|cc}
\hline \hline & & 1992 & 1993 \\
\hline Number of events & $N_{\mathrm{had}}$ & 343731 & 403108 \\
\hline Tagged hemispheres & $N_{\ell}$ & 8455.2 & 9975.9 \\
& $N_{\mathrm{v}}-N_{\overline{\mathrm{v}}}$ & 29488 & 33874 \\
& $N_{\mathrm{a}}-N_{\overline{\mathrm{v}}}$ & 36561.2 & 42297.9 \\
\hline Double-tagged events & $N_{\ell \ell}$ & 186.6 & 246.9 \\
& $N_{\mathrm{vv}}-N_{\mathrm{v} \overline{\mathrm{v}}}+N_{\overline{\mathrm{vv}}}$ & 2645 & 2994 \\
& $N_{\ell \mathrm{v}}-N_{\ell \overline{\mathrm{v}}}$ & 1486.6 & 1738.5 \\
& $N_{\mathrm{aa}}-N_{\mathrm{a} \overline{\mathrm{v}}}+N_{\overline{\mathrm{vv}}}$ & 4018.2 & 4608.5 \\
\hline
\end{tabular}

Table 1: Numbers of tagged hemispheres and double-tagged events in each year of the data. Background in the lepton samples has been subtracted. The numbers of hemispheres tagged by leptons, $N_{\ell}$, is slightly smaller than the sum of those tagged by electrons and muons given in section 5 because hemispheres containing both electrons and muons were counted only once. 


\begin{tabular}{c|cc|cc}
\hline \hline & \multicolumn{2}{|c|}{1992} & \multicolumn{2}{c}{1993} \\
Tag & $\mathbf{c} \overline{\mathbf{c}}$ & $\mathrm{u} \overline{\mathrm{u}}+\mathrm{d} \overline{\mathrm{d}}+\mathrm{s} \overline{\mathrm{s}}$ & $\mathbf{c} \overline{\mathbf{c}}$ & $\mathrm{u} \overline{\mathrm{u}}+\mathrm{d} \overline{\mathrm{d}}+\mathrm{s} \overline{\mathrm{s}}$ \\
\hline$\ell$ & $\mathbf{0 . 3 6 3 \pm \mathbf { 0 . 0 1 3 }}$ & $\mathbf{0 . 0 1 3} \pm \mathbf{0 . 0 0 1}$ & $\mathbf{0 . 3 5 8} \pm \mathbf{0 . 0 1 2}$ & $\mathbf{0 . 0 1 3} \pm \mathbf{0 . 0 0 1}$ \\
$\mathrm{v}-\overline{\mathrm{v}}$ & $1.000 \pm \mathbf{0 . 0 2 7}$ & $\mathbf{0 . 0 9 0} \pm \mathbf{0 . 0 0 8}$ & $\mathbf{0 . 9 8 2} \pm \mathbf{0 . 0 2 8}$ & $\mathbf{0 . 0 8 9} \pm \mathbf{0 . 0 0 9}$ \\
$\mathrm{a}-\overline{\mathrm{v}}$ & $1.364 \pm \mathbf{0 . 0 2 9}$ & $\mathbf{0 . 1 0 3} \pm \mathbf{0 . 0 0 8}$ & $1.340 \pm \mathbf{0 . 0 3 0}$ & $\mathbf{0 . 1 0 1} \pm \mathbf{0 . 0 0 9}$ \\
\hline
\end{tabular}

Table 2: Percentage hemisphere tagging probabilities for light quark events in each year of the data. Errors are due to Monte Carlo statistics.

Table 3 summarises the values of $\Gamma_{\mathrm{b}} / \Gamma_{\text {had }}$ and $\varepsilon^{\mathrm{b}}$ calculated from the numbers of tagged hemispheres and double-tagged events, and the tagging probabilities for light quark events. The values have been corrected for the effect of the hemisphere tagging efficiency correlation. The measurements labelled 'lepton' and 'vertex' are statistically independent measurements obtained using lepton tags $\left(N_{\ell}\right.$ and $\left.N_{\ell \ell}\right)$ or vertex tags $\left(N_{\mathrm{v}}-N_{\overline{\mathrm{v}}}\right.$ and $\left.N_{\mathrm{vv}}-N_{\mathrm{v} \overline{\mathrm{v}}}+N_{\overline{\mathrm{vv}}}\right)$ alone. Events with one hemisphere tagged by a lepton and the other by a vertex, referred to as 'mixed' events, provide a third measurement of $\Gamma_{\mathrm{b} \overline{\mathrm{b}}} / \Gamma_{\mathrm{had}}$. In this case, the combination of $N_{\ell}, N_{\mathrm{v}}-N_{\overline{\mathrm{v}}}$ and $N_{\ell \mathrm{v}}-N_{\ell \overline{\mathrm{v}}}$ is used to determine the three unknowns $\Gamma_{\mathrm{b} \overline{\mathrm{b}}} / \Gamma_{\mathrm{had}}, \varepsilon_{\ell}^{\mathrm{b}}$ and $\varepsilon_{\mathrm{v}}^{\mathrm{b}}-\varepsilon_{\overline{\mathrm{v}}}^{\mathrm{b}}$. Finally, the overall result is given by the combination of $N_{\mathrm{a}}-N_{\overline{\mathrm{v}}}$ and $N_{\mathrm{aa}}-N_{\mathrm{a} \overline{\mathrm{v}}}+N_{\overline{\mathrm{vv}}}$, and includes all the statistics of the above three combinations.

Including the systematic errors discussed in previous sections, the result

$$
\frac{\Gamma_{\mathrm{b} \overline{\mathrm{b}}}}{\Gamma_{\mathrm{had}}}=0.2171 \pm 0.0021 \pm 0.0021
$$

was obtained, where the first error is statistical and the second includes all systematic errors except for the uncertainty from $\Gamma_{\mathrm{c} \bar{c}} / \Gamma_{\text {had }}$. The effect of this uncertainty can be expressed as an explicit dependence on $\Gamma_{\mathrm{c} \overline{\mathrm{c}}} / \Gamma_{\mathrm{had}}$ :

$$
\frac{\Delta \Gamma_{\mathrm{b} \overline{\mathrm{b}}} / \Gamma_{\mathrm{had}}}{\Gamma_{\mathrm{b} \overline{\mathrm{b}}} / \Gamma_{\mathrm{had}}}=-0.086 \frac{\Delta \Gamma_{\mathrm{c} \overline{\mathrm{c}}} / \Gamma_{\mathrm{had}}}{\Gamma_{\mathrm{c} \overline{\mathrm{c}}} / \Gamma_{\mathrm{had}}},
$$

where $\Delta \Gamma_{\mathrm{c}} / \Gamma_{\text {had }}$ is the deviation of $\Gamma_{\mathrm{c} \overline{\mathrm{c}}} / \Gamma_{\text {had }}$ from the value of 0.171 predicted by the Standard Model and used in this analysis. A fractional variation of $\Delta \Gamma_{\mathrm{c} \bar{c}} / \Gamma_{\text {had }}$ of $\pm 8 \%$, corresponding to the present precision of measurements at LEP [3], would result in a variation of $\mp 0.0015$ in $\Gamma_{\mathrm{b} b} / \Gamma_{\text {had }}$. The systematic errors on the measured value of $\Gamma_{\mathrm{b}} / \Gamma_{\text {had }}$ are summarised in table 4 . The error due to Monte Carlo statistics includes those due to the estimation of the tagging probabilities for light quark events

\begin{tabular}{|c|c|c|c|c|}
\hline & Tag & 1992 & 1993 & Average \\
\hline \multirow[t]{4}{*}{$\overline{\Gamma_{\mathrm{b} \overline{\mathrm{b}}} / \Gamma_{\mathrm{had}}}$} & lepton & $0.2512 \pm 0.0187$ & $0.2259 \pm 0.0148$ & $0.2375 \pm 0.0118$ \\
\hline & vertex & $0.2169 \pm 0.0037$ & $0.2161 \pm 0.0035$ & $0.2165 \pm 0.0025$ \\
\hline & mixed & $0.2189 \pm 0.0054$ & $0.2166 \pm 0.0052$ & $0.2177 \pm 0.0037$ \\
\hline & overall & $0.2175 \pm 0.0030$ & $0.2167 \pm 0.0029$ & $0.2171 \pm 0.0021$ \\
\hline \multirow[t]{5}{*}{$\varepsilon^{\mathrm{b}}$} & lepton & $0.0462 \pm 0.0035$ & $0.0517 \pm 0.0034$ & $0.0492 \pm 0.0024$ \\
\hline & lepton (mixed) & $0.0530 \pm 0.0014$ & $0.0540 \pm 0.0013$ & $0.0535 \pm 0.0010$ \\
\hline & vertex & $0.1873 \pm 0.0033$ & $0.1841 \pm 0.0031$ & $0.1856 \pm 0.0022$ \\
\hline & vertex (mixed) & $0.1856 \pm 0.0047$ & $0.1837 \pm 0.0044$ & $0.1846 \pm 0.0032$ \\
\hline & overall & $0.2309 \pm 0.0032$ & $0.2286 \pm 0.0030$ & $0.2297 \pm 0.0022$ \\
\hline
\end{tabular}
and of the muon fake rate.

Table 3: Values of $\Gamma_{\mathrm{b}} / \Gamma_{\text {had }}$ and $\varepsilon^{\mathrm{b}}$ after correlation correction. The efficiencies marked 'mixed' were obtained from the mixed-tagged events. Errors are only statistical. 


\begin{tabular}{|c|c|}
\hline Source & Error \\
\hline Electron detection efficiency & \pm 0.00012 \\
\hline Muon detection efficiency & \pm 0.00005 \\
\hline Photon conversion & \pm 0.00027 \\
\hline Hadronic bg. in electron ID & \pm 0.00027 \\
\hline Hadronic bg. in muon ID & \pm 0.00041 \\
\hline Tracking resolution & \pm 0.00071 \\
\hline c quark fragmentation & \pm 0.00072 \\
\hline Charmed hadron fractions & $\pm \mathbf{0 . 0 0 0 8 9}$ \\
\hline Charmed hadron lifetimes & \pm 0.00040 \\
\hline Branching fraction $B(\mathrm{c} \rightarrow \ell)$ & $\pm \mathbf{0 . 0 0 0 2 8}$ \\
\hline Charm decay modelling & \pm 0.00024 \\
\hline Charm decay charged multiplicity & \pm 0.00073 \\
\hline Branching fraction $B\left(\mathrm{D} \rightarrow \mathrm{K}^{0}\right)$ & \pm 0.00057 \\
\hline $\mathrm{b} / \mathrm{c}$ quark production in $\mathrm{u} \overline{\mathrm{u}}, \mathrm{d} \overline{\mathrm{d}}, \mathrm{s} \overline{\mathrm{s}}$ events & $\pm \mathbf{0 . 0 0 0 4 8}$ \\
\hline Inclusive $\mathrm{K}^{0}$ production & \pm 0.00028 \\
\hline Inclusive hyperon production & \pm 0.00017 \\
\hline Efficiency correlation & \pm 0.00069 \\
\hline Event Selection & \pm 0.00025 \\
\hline Monte Carlo statistics & $\pm \mathbf{0 . 0 0 0 4 9}$ \\
\hline Total & \pm 0.00210 \\
\hline
\end{tabular}

Table 4: Systematic errors on the measured value of $\Gamma_{\mathrm{b}} / \Gamma_{\mathrm{had}}$.

The transverse momentum cuts for electrons and muons and the decay length significance cuts were varied within $\pm 0.2 \mathrm{GeV} / c$ and \pm 2 respectively. The values of $\Gamma_{\mathrm{b}} / \Gamma_{\mathrm{had}}$ obtained using different cuts are shown in figure 3 . The changes in the result are consistent with the expected fluctuations due to the independent part of the data statistics.

An independent measurement of $\Gamma_{\mathrm{b}} / \Gamma_{\text {had }}$ using single and double lepton tagged events was performed over an extended $\cos \theta$ range and with lower $p$ and $p_{t}$ cuts. No restrictions on the thrust value or thrust axis direction were imposed, and the lepton identification requirements were modified. In particular, the electron selection was extended beyond $|\cos \theta|=0.715$ using variables described in previous publications [28]. Extending the acceptance in this way improved the precision of the lepton-only analysis, at the expense of increased efficiency correlations. These were estimated using Monte Carlo simulation for the main geometrical and kinematic correlations, and data for the details of the lepton identification. The results obtained were consistent with those presented here.

The measured value of $\Gamma_{\mathrm{b} b} / \Gamma_{\text {had }}$ presented here is calculated from the ratio of cross sections $\sigma\left(\mathrm{e}^{+} \mathrm{e}^{-} \rightarrow \mathrm{b} \overline{\mathrm{b}}\right) / \sigma\left(\mathrm{e}^{+} \mathrm{e}^{-} \rightarrow\right.$ hadrons $)$, overwhelmingly dominated by $\mathrm{Z}^{0}$ decays. The effects of photon exchange are predicted by ZFITTER [2] to reduce the measured ratio by 0.0003 compared with that expected from pure $Z^{0}$ exchange. This effect has been neglected in this paper.

A substantial fraction of the data collected in 1993 was taken at centre-of-mass energies $E_{\mathrm{cm}}$ above or below the peak of the $\mathrm{Z}^{0}$ resonance. Of the total of 403108 events, 49208 events were recorded at $E_{\mathrm{cm}}=89.45 \mathrm{GeV}$, and 71024 events at $93.04 \mathrm{GeV}$. These off-peak data are expected to have almost the same $\mathrm{b} \bar{b}$ fraction, as the hadronic cross sections at these energies are still dominated by $\mathrm{Z}^{0}$ decays. The off-peak data were therefore analysed together with the 1993 on-peak data as a single sample. Figure 4 compares the values of $\Gamma_{\mathrm{b}} / \Gamma_{\text {had }}$ obtained by analysing the on-peak and off-peak data separately. The expected $E_{\mathrm{cm}}$ dependence of the $\mathrm{b} \overline{\mathrm{b}}$ fraction in the hadronic cross section, calculated 
using the ZFITTER program [2], is also shown. The results are consistent within the statistical errors.

The reproducibility of the method was checked by analysing the Monte Carlo events that were used in the estimation of the light quark efficiencies and the muon background. The sample contained 573767 events passing the event selection, of which 124604 were $b \bar{b}$ events. The analysis gave $a$ result of $\Gamma_{\mathrm{b}} / \Gamma_{\text {had }}=0.2181 \pm 0.0027$ where the error is statistical. The result has been corrected for the tagging efficiency correlation between hemispheres. The result is consistent with the input value $0.2172 \pm 0.0005$. The measured tagging efficiency $\varepsilon^{\mathrm{b}}=0.2124 \pm 0.0025$ is also consistent with the true efficiency $0.2143 \pm 0.0010$. The efficiency in the Monte Carlo sample is significantly lower than that in the data because of the short bottom hadron lifetime (1.4ps) and low bottom hadron mean charged decay multiplicity used in the simulation.

The result presented here is consistent with, and significantly more precise than, previously published OPAL measurements of $\Gamma_{\mathrm{b}} / \Gamma_{\text {had }}[26,27,29,30]$ based on earlier data. Making reasonable assumptions about error correlations, the present analysis would carry a $93 \%$ weight if averaged with these earlier results. However, the treatment of systematic errors has been refined since those publications, making it difficult to combine them correctly with the new measurement. The result of the present analysis therefore supersedes the previous OPAL measurements.

\section{Summary and Conclusion}

The fraction of $\mathrm{Z}^{0} \rightarrow \mathrm{b} \overline{\mathrm{b}}$ events in hadronic $\mathrm{Z}^{0}$ decays was measured using the data collected by OPAL in 1992 and 1993, giving a result of

$$
\frac{\Gamma_{\mathrm{b} \overline{\mathrm{b}}}}{\Gamma_{\mathrm{had}}}=0.2171 \pm 0.0021 \pm 0.0021
$$

where the first error is statistical and the second systematic. The systematic error does not include the effects of the uncertainty in $\Gamma_{c \bar{c}} / \Gamma_{\text {had }}$. The result depends on $\Gamma_{c \bar{c}} / \Gamma_{\text {had }}$ as follows:

$$
\frac{\Delta \Gamma_{\mathrm{b} \overline{\mathrm{b}}} / \Gamma_{\mathrm{had}}}{\Gamma_{\mathrm{b} \overline{\mathrm{b}}} / \Gamma_{\mathrm{had}}}=-0.086 \frac{\Delta \Gamma_{\mathrm{c} \overline{\mathrm{c}}} / \Gamma_{\mathrm{had}}}{\Gamma_{\mathrm{c} \overline{\mathrm{c}}} / \Gamma_{\mathrm{had}}},
$$

where $\Delta \Gamma_{\mathrm{c}} / \Gamma_{\text {had }}$ is the deviation of $\Gamma_{\mathrm{c} \bar{c}} / \Gamma_{\text {had }}$ from the value 0.171 predicted by the Standard Model. The total error excluding the $\Gamma_{\mathrm{c} \overline{\mathrm{c}}} / \Gamma_{\mathrm{had}}$ dependence is $\pm 1.36 \%$ of the measurement. The result is consistent with other recently published measurements of $\Gamma_{\mathrm{b}} / \Gamma_{\mathrm{had}}$ made by the LEP Collaborations [31,32] and at the SLC [33]. The result is slightly lower than the most precise previously published measurement, from ALEPH [31], of $0.2193 \pm 0.0029$ for $\Gamma_{\mathrm{c} \bar{c}} / \Gamma_{\text {had }}=0.171$, and is of a similar precision.

The measured value of $\Gamma_{\mathrm{b}} / \Gamma_{\text {had }}$ is compared with the Standard Model prediction, obtained using the ZFITTER program [2], in figure 5 . The value of $\Gamma_{\mathrm{d}} / \Gamma_{\text {had }}$ predicted by the Standard Model is also shown for comparison. The result is consistent within one standard deviation with the Standard Model prediction for top quark masses smaller than $189 \mathrm{GeV} / c^{2}$. 


\section{Acknowledgements}

It is a pleasure to thank the SL Division for the efficient operation of the LEP accelerator, the precise information on the absolute energy, and their continuing close cooperation with our experimental group. In addition to the support staff at our own institutions we are pleased to acknowledge the Department of Energy, USA,

National Science Foundation, USA,

Particle Physics and Astronomy Research Council, UK,

Natural Sciences and Engineering Research Council, Canada,

Fussefeld Foundation,

Israel Ministry of Science,

Israel Science Foundation, administered by the Israel Academy of Science and Humanities,

Minerva Gesellschaft,

Japanese Ministry of Education, Science and Culture (the Monbusho) and a grant under the Monbusho International Science Research Program,

German Israeli Bi-national Science Foundation (GIF),

Direction des Sciences de la Matière du Commissariat à l'Energie Atomique, France,

Bundesministerium für Forschung und Technologie, Germany,

National Research Council of Canada,

A.P. Sloan Foundation and Junta Nacional de Investigação Científica e Tecnológica, Portugal. 


\section{References}

[1] See, for example,

J.H. Kühn, P.M. Zerwas, in: Z Physics at LEP 1, eds. G. Altarelli, R. Kleiss and C. Verzegnassi, vol. 1 (CERN 89-08, 1989) pp. 271-275.

[2] Calculated using the ZFITTER program described in:

D. Bardin et al., CERN-TH 6443/92 (May 1992); Phys. Lett. B255 (1991) 290; Nucl. Phys. B351 (1991) 1; Z. Phys. C44 (1989) 493.

[3] The LEP Collaborations, ALEPH, DELPHI, L3, OPAL and the LEP Electroweak Working Group, Updated Parameters of the $\mathrm{Z}^{0}$ Resonance from Combined Preliminary Data of the LEP Experiments, CERN PPE/93-157.

[4] OPAL Collab., K. Ahmet et al., Nucl. Instrum. Methods A305 (1991) 275.

[5] P.P. Allport et al., Nucl. Instrum. Methods A324 (1993) 34.

[6] P.P. Allport et al., CERN-PPE/94-16, Submitted to Nucl. Instrum. Methods.

[7] M. Hauschild et al., Nucl. Instrum. Methods A314 (1992) 74;

O. Biebel et al., Nucl. Instrum. Methods A323 (1992) 169.

[8] OPAL Collab., G. Alexander et al., Z. Phys. C52 (1991) 175.

[9] JADE Collab., W. Bartel et al., Z. Phys. C33 (1986) 23;

JADE Collab., S. Bethke et al., Phys. Lett. B213 (1988) 235.

[10] OPAL Collab., M.Z. Akrawy et al., Z. Phys. C49 (1991) 375.

[11] T. Sjöstrand, PYTHIA 5.6 and JETSET 7.3: Physics and Manual, CERN-TH.6488/92;

T. Sjöstrand and M. Bengtsson, Computer Physics Commun. 43 (1987) 367;

T. Sjöstrand, Computer Physics Commun. 39 (1986) 347.

[12] J. Allison et al., Nucl. Instrum. Methods A317 (1992) 47.

[13] OPAL Collab., M.Z. Akrawy et al., Z. Phys. C47 (1990) 505.

[14] C. Peterson, D. Schlatter, I. Schmitt and P. Zerwas, Phys. Rev. D27 (1983) 105.

[15] ALEPH Collab., D. Decamp et al., Phys. Lett. B244 (1990) 551;

L3 Collab., B. Adeva et al., Phys. Lett. B261 (1991) 177;

OPAL Collab., M.Z. Akrawy et al., Phys. Lett. B263 (1991) 311;

ALEPH Collab., D. Decamp et al., Phys. Lett. B266 (1991) 218;

DELPHI Collab., P. Abreu et al., Z. Phys. C56 (1992) 47.

[16] CELLO Collab., H.J. Behrend et al., Z. Phys. C19 (1983) 291;

MARKJ Collab., B. Adeva et al., Phys. Rev. Lett. 51 (1983) 443;

TASSO Collab., M. Althoff et al., Phys. Lett. B146 (1984) 443;

TASSO Collab., M. Althoff et al., Z. Phys. C22 (1984) 219;

TPC Collab., H. Aihara et al., Z. Phys. C27 (1985) 39;

DELCO Collab., T. Pal et al., Phys. Rev. D33 (1986) 2708;

JADE Collab., W. Bartel et al., Z. Phys. C33 (1987) 339;

MARK II Collab., R.A. Ong et al., Phys. Rev. Lett. 60 (1988) 2587;

ARGUS Collab., H. Albrecht et al., Phys. Lett. B278 (1992) 202. 
[17] G. Altarelli et al., Nucl. Phys. B208 (1982) 365.

[18] DELCO Collab., W. Bacino et al., Phys. Rev. Lett. 43 (1979) 1073.

[19] MARK III Collab., R.M. Baltrusaitis et al., Phys. Rev. Lett. 54 (1985) 1976.

[20] ARGUS Collab., H. Albrecht et al., Z. Phys. C52 (1991) 353;

CLEO Collab., D. Bortoletto et al., Phys. Rev. D37 (1988) 1719.

[21] Particle Data Group, K. Hikasa et al., Phys. Rev. D45 (1992) S1.

[22] MARK III collab., D. Coffman et al., Phys. Lett. B263 (1991) 135.

[23] M.H. Seymour, LU TP 94-7, Lund Univ., May 1994.

[24] OPAL Collab., G. Alexander et al., Phys. Lett. B264 (1991) 467.

[25] OPAL Collab., P.D. Acton et al., Phys. Lett. B291 (1992) 503.

[26] OPAL Collab., R. Akers et al., Z. Phys. C60 (1993) 199.

[27] OPAL Collab., P.D. Acton et al., Z. Phys. C58 (1993) 523.

[28] OPAL Collab., P.D. Acton et al., Z. Phys. C60 (1993) 19.

[29] OPAL Collab., P.D. Acton et al., Z. Phys. C60 (1993) 579.

[30] OPAL Collab., R. Akers et al., Z. Phys. C61 (1994) 357.

[31] ALEPH Collab., D. Buskulic et al., Phys. Lett. B313 (1993) 535.

[32] ALEPH Collab., D. Buskulic et al., CERN-PPE/94-017;

ALEPH Collab., D. Buskulic et al., Phys. Lett. B313 (1993) 549;

DELPHI Collab., P. Abreu et al., Phys. Lett. B295 (1992) 383;

DELPHI Collab., P. Abreu et al., Z. Phys. C56 (1992) 47;

DELPHI Collab., P. Abreu et al., Phys. Lett. B281 (1992) 383;

L3 Collab., O. Adriani et al., Phys. Lett. B307 (1993) 237;

L3 Collab., B. Adeva et al., Phys. Lett. B261 (1991) 177.

[33] Mark II Collab., R. Jacobsen et al., Phys. Rev. Lett. 67 (1991) 3347;

Mark II Collab., J.F. Kral et al., Phys. Rev. Lett. 64 (1990) 1211.

[34] OPAL Collab., P.D. Acton et al., Z. Phys. C60 (1993) 217.

[35] OPAL Collab., R. Akers et al., Z. Phys. C61 (1994) 209. 

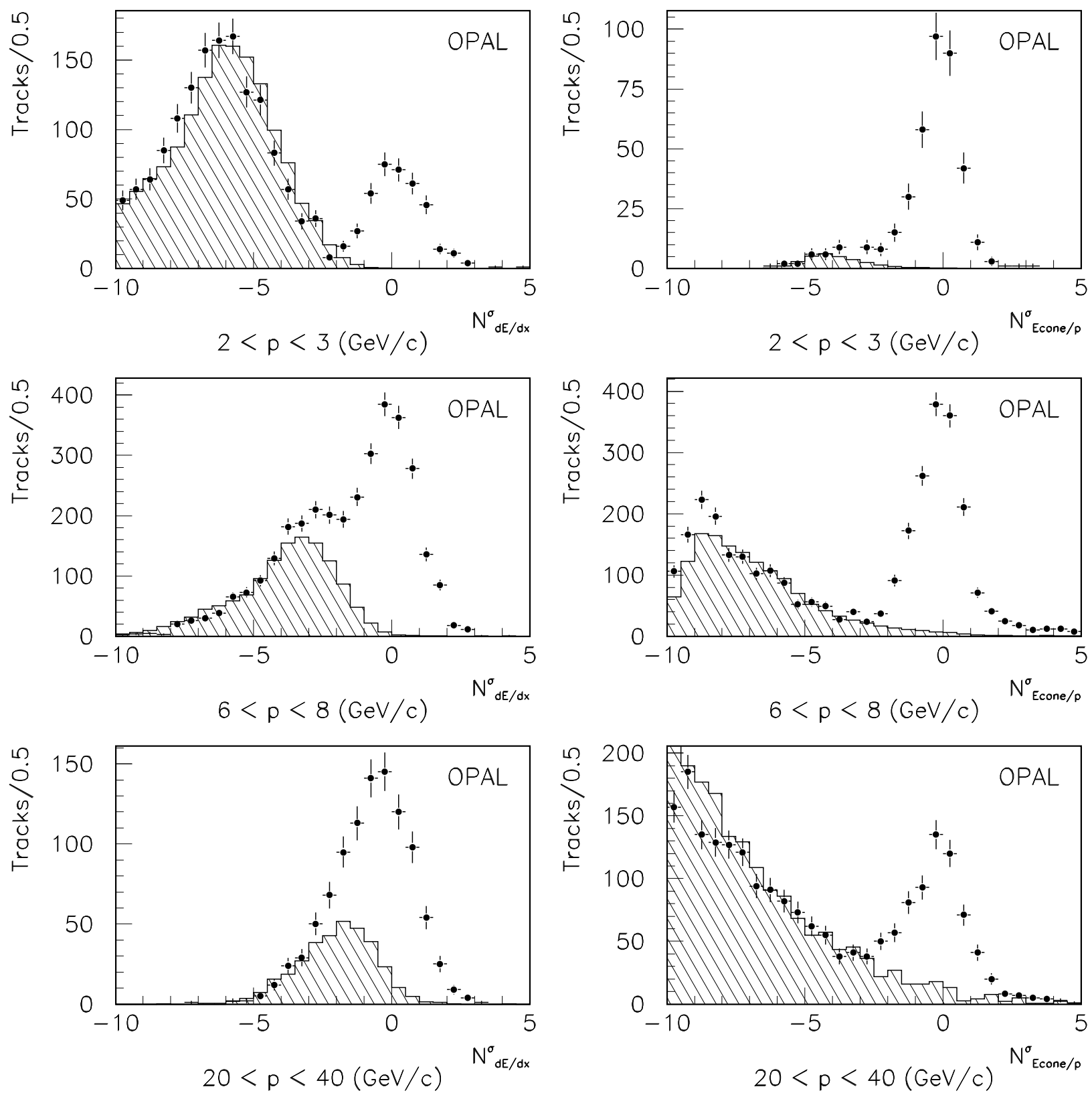

Figure 1: Distributions of $N_{\mathrm{d} E / \mathrm{d} x}^{\sigma}$ (left column) and $N_{E_{\text {cone }} / p}^{\sigma}$ (right column) for three momentum ranges of tracks passing all other electron identification selection cuts. Shaded histograms indicate background estimated using anti-cut samples with $-7<N_{E_{\text {cone }} / p}^{\sigma}<-3$ (left column) or with $-3<N_{\mathrm{d} E / \mathrm{d} x}^{\sigma}<-2$ (right column). 


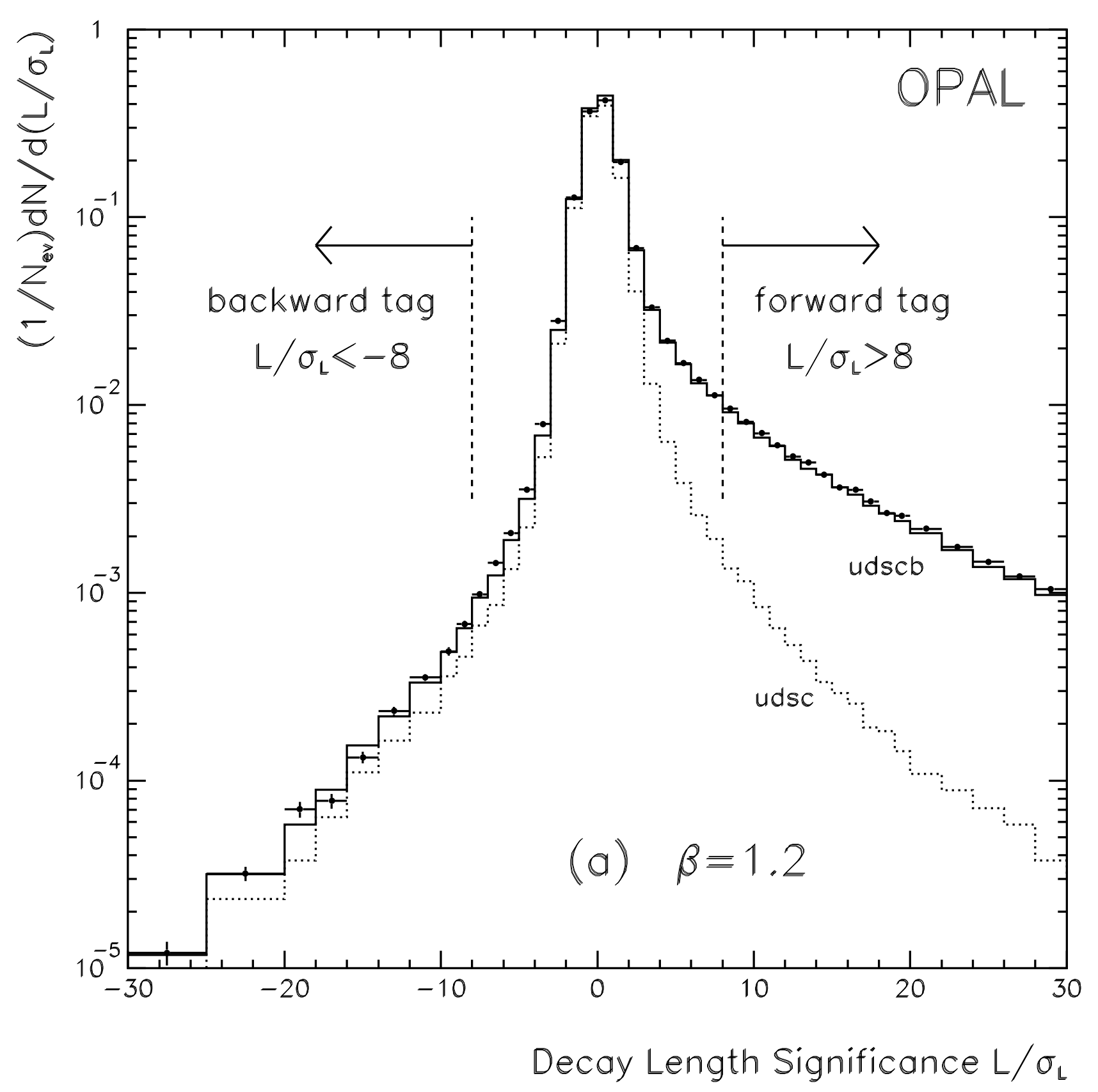

Figure 2: Comparison of $L / \sigma_{L}$ distributions from data (points with error bars) and Monte Carlo (full histograms). As discussed in the text, the most important region of the plot for this analysis is for backward decay length significances around the backward tag cut. Dotted histograms indicate contributions from light flavour events. Smearing has been applied to the Monte Carlo events as described in the text with smearing factors (a) $\beta=1.2$ and (b) $\beta=1.4$. The average bottom hadron lifetime and mean bottom hadron charged decay multiplicity have been adjusted to OPAL measured values $[34,35]$ for this plot. 


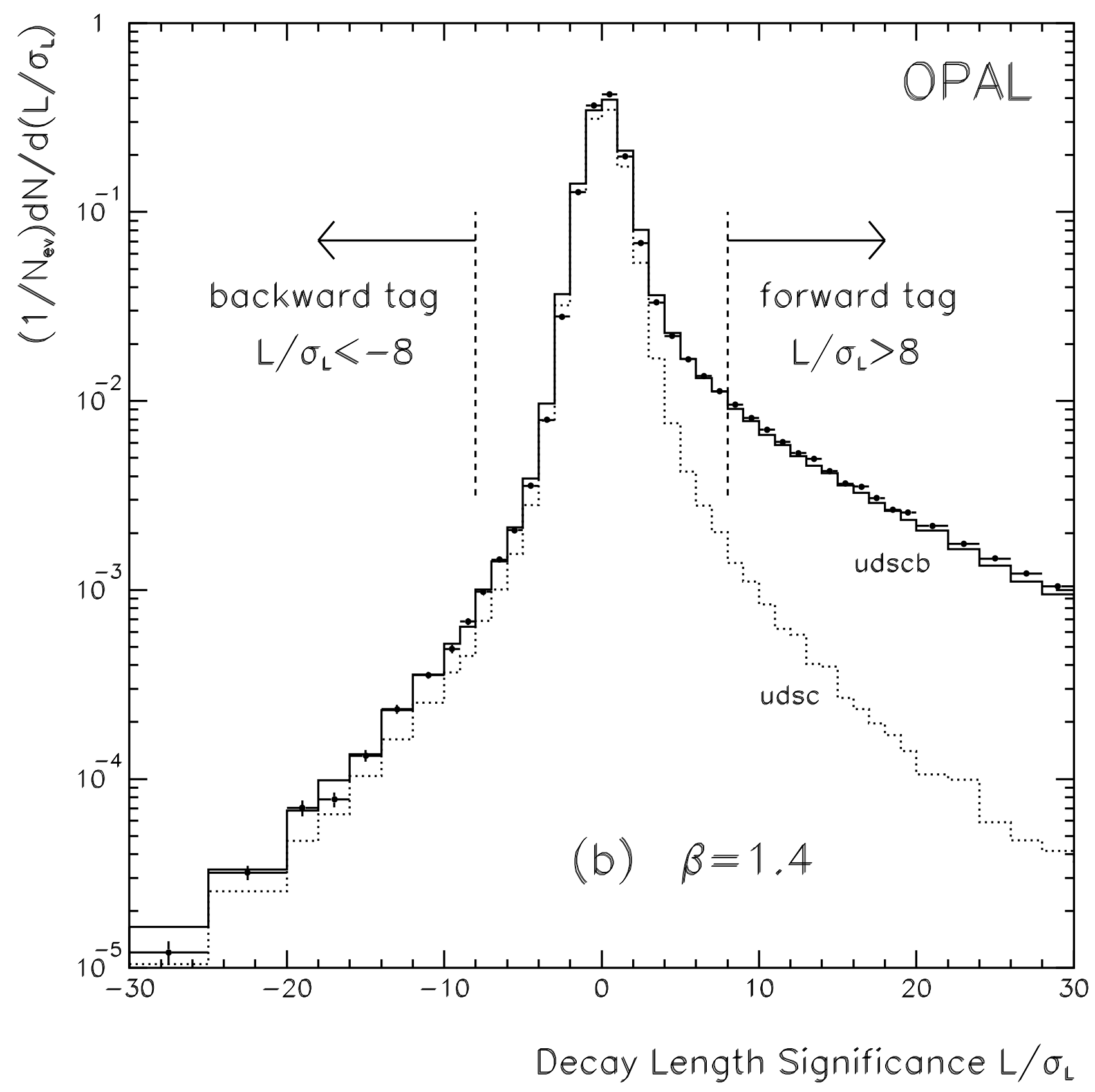



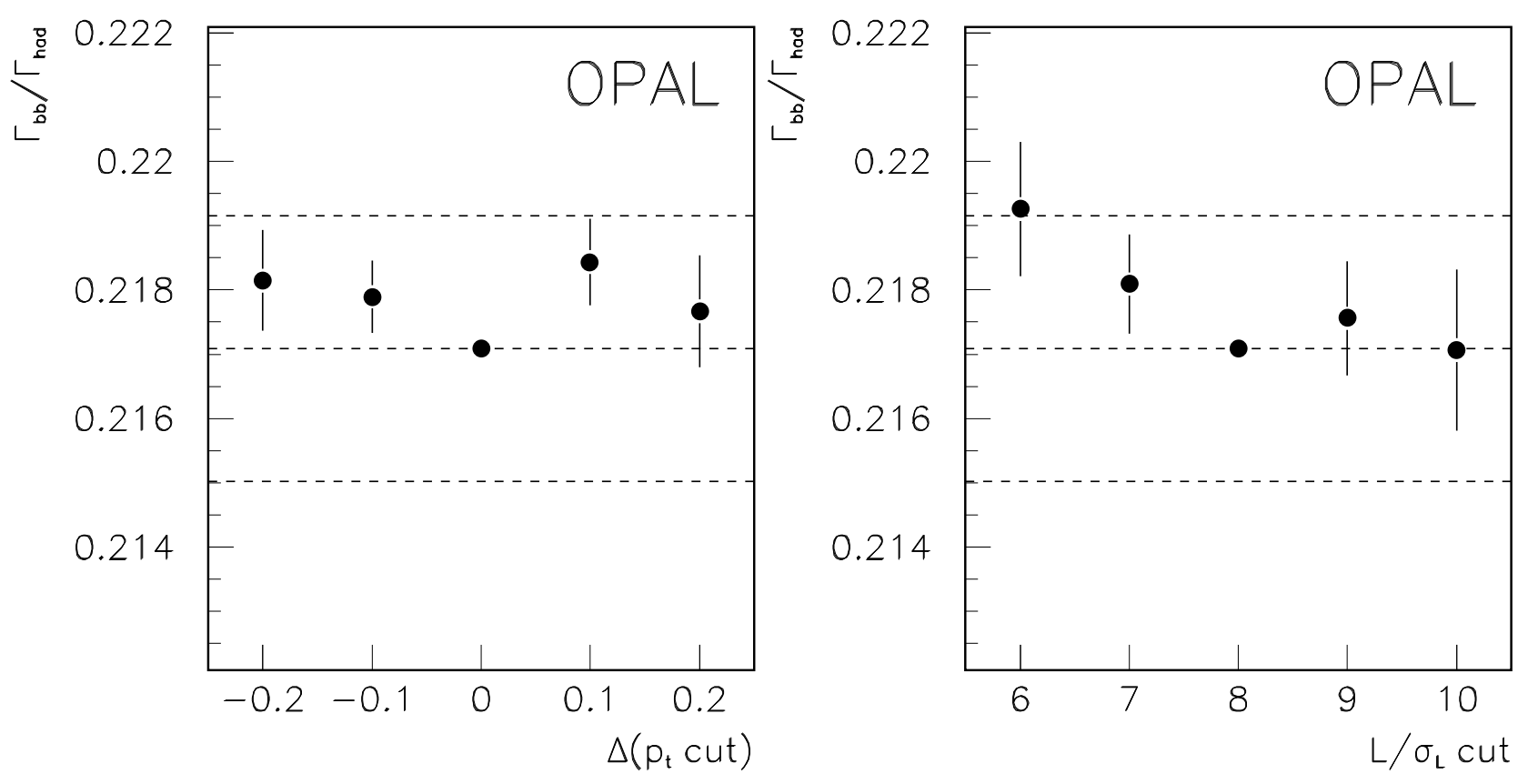

Figure 3: Values of $\Gamma_{\mathrm{b}} / \Gamma_{\text {had }}$ obtained using different cuts on the lepton transverse momentum $p_{t}$ and the decay length significance $L / \sigma_{L}$. The results have been corrected for hemisphere tagging efficiency correlation. Dashed lines indicate the central value and its statistical error. Error bars are the statistical errors on the differences from the central result. 


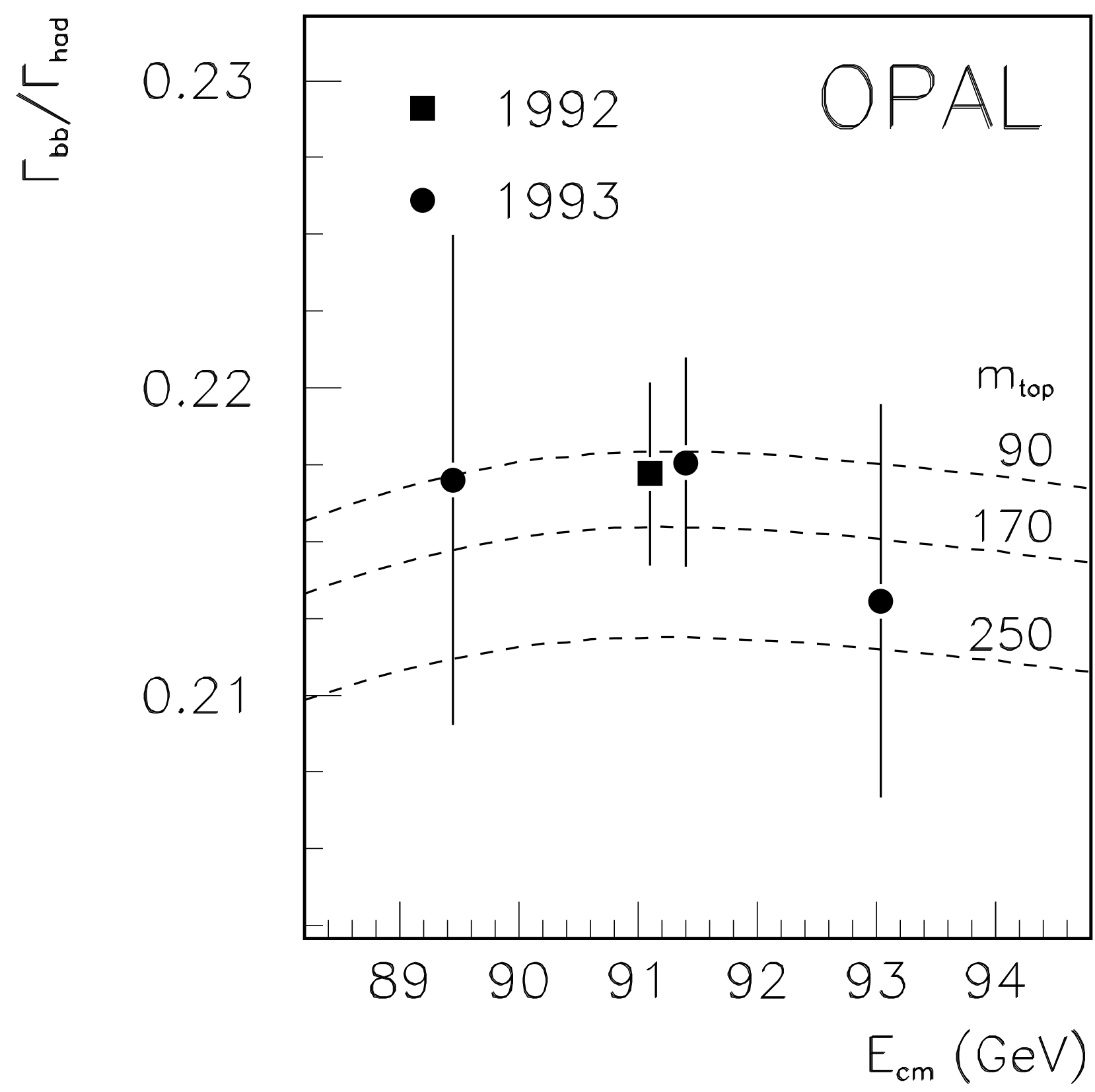

Figure 4: Values of $\Gamma_{\mathrm{b}} / \Gamma_{\text {had }}$ obtained from data samples taken at different centre-of-mass energies $E_{\mathrm{cm}}$. Errors are statistical. Points for 1992 and 1993 on-peak data are slightly shifted in $E_{\mathrm{cm}}$. Dashed lines indicate the Standard Model prediction of the ratio $\sigma\left(\mathrm{e}^{+} \mathrm{e}^{-} \rightarrow \mathrm{b} \overline{\mathrm{b}}\right) / \sigma\left(\mathrm{e}^{+} \mathrm{e}^{-} \rightarrow\right.$ hadrons $)$ obtained using the ZFITTER program for top quark masses of 90,170 and $250 \mathrm{GeV} / c^{2}$, respectively from upper to lower. A value of $m_{\mathrm{Higgs}}=300 \mathrm{GeV} / c^{2}$ is assumed. 


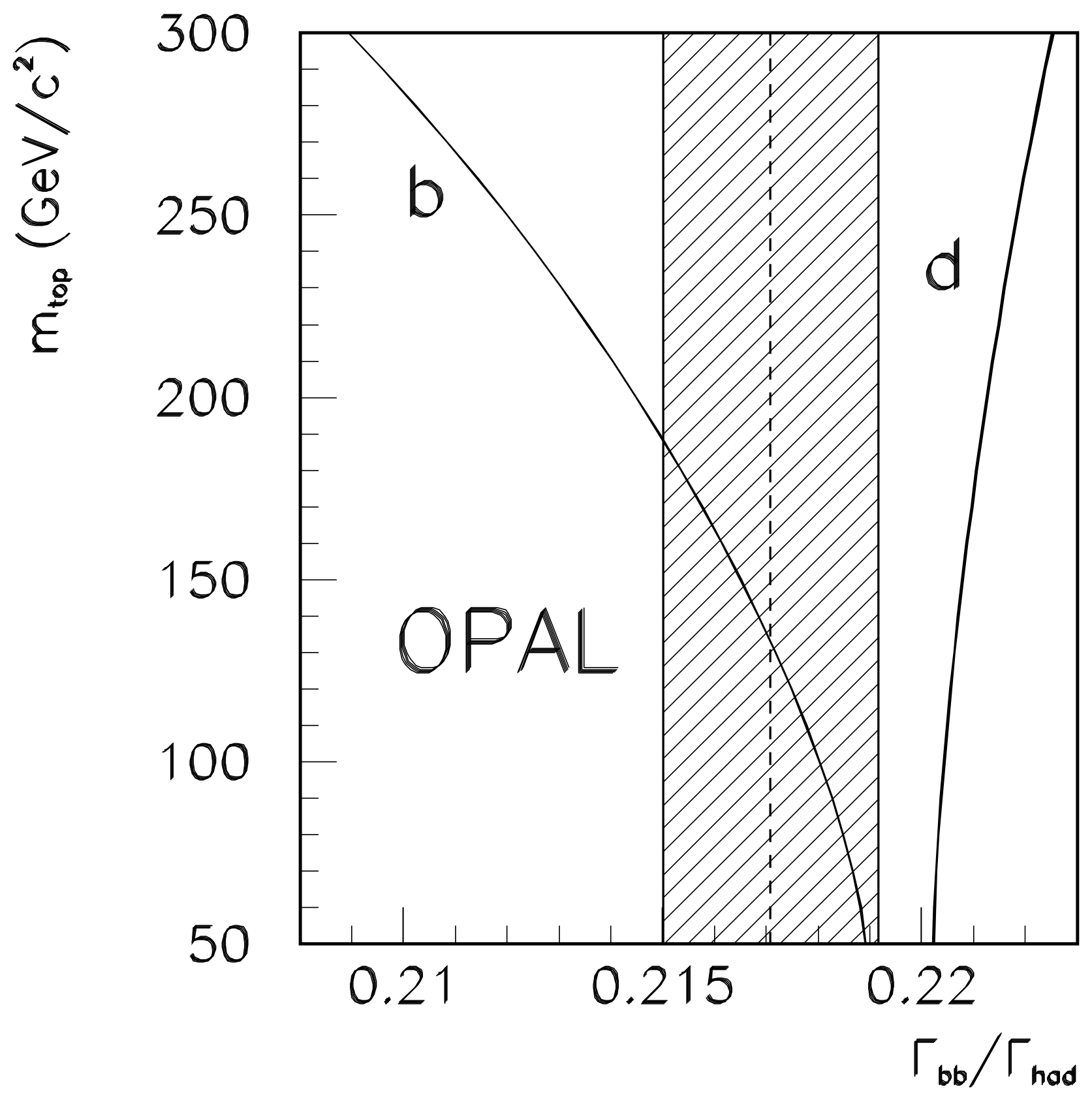

Figure 5: Comparison between the measured value of $\Gamma_{\mathrm{b}} / \Gamma_{\mathrm{had}}$ and the Standard Model prediction obtained using the ZFITTER program. The hatched area shows the plus-or-minus one standard deviation range of this measurement. Curves indicate the predicted values of $\Gamma_{\mathrm{b}} / \Gamma_{\text {had }}$ (left) and $\Gamma_{d \bar{d}} / \Gamma_{\text {had }}$ (right) as functions of the top quark mass $m_{\mathrm{top}}$. The widths of the curves represent the uncertainty due to Higgs particle masses in the range $60-1000 \mathrm{GeV} / c^{2}$. 